\title{
Notation systems for infinitary derivations
}

\author{
Wilfried Buchholz \\ Mathematisches Institut der Universität München, Theresienstrasse 39, W-8000 München 2, \\ Federal Republic of Germany \\ Dedicated to Kurt Schütte on the occasion of his 80 th birthday
}

Received September 26, 1989

It is one of Kurt Schütte's great merits to have established cut-elimination on infinitary derivations as a powerful and elegant tool for proof-theoretic investigations. Compared to the Gentzen-Takeuti approach where ordinals are assigned to finite derivations in a rather cryptic way, the use of infinitary derivations together with the canonical assignment of ordinals as lengths of derivations provides a very perspicious and conceptually clear-cut method which has proved successful even with respect to the strongest systems analyzed till now. But on the other side something is lost when passing from finite to (unrestricted) infinite derivations, in so far as along these lines one only obtains information on the provable $\Pi_{1}^{1}$-sentences of a formal theory, while Gentzen's method - if successfully applied - yields stronger results, e.g. bounds for provable $\Pi_{2}^{0}$-sentences (provably recursive functions) or the unprovability of primitive recursive wellfoundedness $P R W O$. Of course, as pointed out by Kreisel [7] such stronger results can be recaptured by arithmetizing the cut-elimination procedure for (primitive) recursively represented infinite derivations via the (Primitive) Recursion Theorem (cf. Schwichtenberg [15], Girard [5]). But this requires a lot of cumbersome and boring coding machinery which on the other side is not completely trivial, and it seems to me that all presentations of this subject in the existing literature are more or less unsatisfactory.

Our purpose here is to provide a technically smooth method for the finitary treatment of infinite derivations in $\omega$-arithmetic $Z^{\infty}$, where we don't need numerical codes but instead are working with natural notations for infinite derivations. These notations are finite terms generated from finite derivations (considered as constants) by certain function symbols $I_{k, A}, R_{C}, E$ corresponding to the operations $\mathscr{I}_{k, A}: Z^{\infty} \rightarrow Z^{\infty}, \mathscr{R}_{C}: Z^{\infty} \times Z^{\infty} \rightarrow Z^{\infty}, \mathscr{E}: Z^{\infty} \rightarrow Z^{\infty}$ which make up the cut-elimination procedure for $Z^{\infty}$ developed by Schütte [12] and Minc [10]. (Minc' contribution was to modify Schütte's cut-elimination procedure by incorporating the so-called repetition-rule, which is crucial for the subsequent work.) 
In order to demonstrate the working of our method we will prove two wellknown results of classical proof theory for the system $Z+T I_{<1}$ (i.e. PeanoArithmetic together with the scheme of transfinite induction along any proper segment of some prim. rec. wellordering $\prec$ ). These results are

(I) If $Z+T I_{<1} \vdash \forall x \exists y R(x, y)\left(R \in \Sigma_{1}^{0}\right)$ then there are prim. rec. functions $\mathrm{g}$, $o: \mathbb{N}^{2} \rightarrow \mathbb{N}$, such that $\min \{m: R(n, m)\}=g(n, \min \{k: o(n, k+1) \nless o(n, k)\})$, for all $n \in \mathbb{N}$.

(II) $P R A \vdash P R W O(\prec) \rightarrow \Pi_{2}^{0}$-Reflection $\left(Z+T I_{<1}\right)$.

Of course in the proof of (I) and (II) we cannot completely dispense with coding. But we only need the comparatively trivial coding of syntactic objects (such as formulas, sequents, finite derivations, etc.) and even this plays a rather marginal rôle, while the central part of our proof is coding-free.

\section{Content}

Section 1 contains besides some preliminary definitions and abbreviations a precise definition of the set $Z_{<}$of all $\left(Z+T I_{<\uparrow}\right)$-derivations. In Sect. 2 we introduce the set $Z^{\infty}$ of derivations of $\omega$-arithmetic and define the Schütte-Minc operator $\mathscr{E}: Z^{\infty} \rightarrow Z^{\infty}$ by transfinite recursion on wellfounded trees. The effect of $\mathscr{E}$ is to lower the cutrank $\operatorname{deg}(\varphi)$ of each $\varphi \in Z^{\infty}$ (with $\left.0<\operatorname{deg}(\varphi)<\omega\right)$ at least by 1 . In [10] $\mathscr{E}$ is denoted $\mathscr{R}_{1}$. In fact the material of this section is not necessary for the proof of (I), (II) above; it only serves as a semantical basis for the syntactic definitions of Sect. 3. In Sect. 3 we define the notation system $Z_{\prec}^{*}$ which contains notations for all $\varphi \in Z^{\infty}$ arising from finite derivations $d \in Z_{\prec}$ via embedding ${ }^{\infty}: Z_{\prec} \rightarrow Z^{\infty}$ and subsequent cut-elimination in $Z^{\infty}$. The main point is that (by a simple recursion on the built up of terms) from every notation $h \in Z_{<}^{*}$ one can compute the endsequent of $\mathbf{v}(h)$ (the $Z^{\infty}$-derivation denoted by $h$ ) and notations $\mathfrak{s}_{0} h, \mathfrak{s}_{1} h, \ldots$ for the immediate subderivations of $\mathbf{v}(h)$. In Sect. 4 we prove (I) and (II) using the work of Sect. 3. In Sect. 5 we generalize the approach of Sect. 3 and introduce the notion of an arbitrary notation system for $\omega$-derivations. This is then used in Sect. 6 to give an alternative description of Minc's continuous cutelimination operator $\mathscr{E}^{\prime}$ for arbitrary (not necessarily wellfounded) proof-figures of $\omega$-arithmetic. In fact $\mathscr{E}$ is an extension of $\mathscr{E}$.

\section{Remarks}

1. We want to emphasize that the present paper has profited considerably by previous presentations of the subject by Girard [5], Minc [10], Schwichtenberg [15]. In some sense it may be considered as a supplement to those; but nevertheless it is completely selfcontained.

2. The idea of using terms (built up from constants and function symbols with a welldefined semantical meaning) as notations for infinite derivations is nothing more than a slight (and rather obvious) generalization of Schütte's approach to systems of ordinal notations as presented in Chap. V of his "Proof Theory" [14].

3. We are indebted to G. Minc, W. Pohlers, and W. Sieg for substantial comments on an earlier version of this paper. 


\section{The formal system $Z_{\prec}$}

\section{Preliminaries}

\subsection{Syntax}

In the following $L$ denotes a fixed $1^{\text {st }}$-order language consisting of the following symbols: a constant 0 (zero), a unary function constant ' (successor), and some predicate symbols. We distinguish two sorts of individual variables, free variables (denoted by $u, v$ ) and bound variables (denoted by $x, y$ ). The closed $L$-terms, $0,0^{\prime}$, $0^{\prime \prime}, \ldots$ are called numerals; we identify numerals and natural numbers and denote them by $i, j, k, m, n$. An expression of the shape $p t_{1} \ldots t_{n}$ or $\sim p t_{1} \ldots t_{n}$, where $p$ is a $n$-ary predicate symbol of $L$ and $t_{1}, \ldots, t_{n}$ are arbitrary $L$-terms, is called a prime formula. Formulas are built up from prime formulas by means of $\wedge, \vee, \forall x, \exists x$. The negation $\neg A$ of a formula $A$ is defined by

$$
\begin{aligned}
& \neg p \vec{t}: \equiv \sim \vec{t}, \quad \neg(\sim p \vec{t}): \equiv p \vec{t}, \quad \neg(A \wedge B): \equiv(\neg A)_{\wedge}^{\vee}(\neg B), \\
& \neg{ }_{\exists}^{\forall} x F(x): \equiv{ }_{\forall}^{\exists} x \neg F(x) .
\end{aligned}
$$

The length $\ell(A)$ of a formula $A$ is defined by

$$
\begin{gathered}
\ell(p \vec{t}): \equiv \ell(\sim \vec{t}):=0, \quad \ell(A \wedge B):=\ell(A \vee B):=\max \{\ell(A), \ell(B)\}+1, \\
\ell(\forall x F(x)):=\ell(\exists x F(x)):=\ell(F(x))+1 .
\end{gathered}
$$

Note that $\ell(\neg A)=\ell(A)$ and $\ell(A(x))=\ell(A(t))$.

In the whole paper we are working with Tait's sequent calculus (cf. [17]), where sequents are finite sets of formulas denoted by $\Gamma, \Delta, \ldots$. The intended meaning of a sequent $\left\{A_{1}, \ldots, A_{k}\right\}$ is the disjunction $A_{1} \vee \ldots \vee A_{k}$. We use the following syntactical variables: $A, B, C$ for formulas, $F$ for 1-place nominal forms (in the sense of Schütte [14]), $\Gamma, \Delta$ for sequents, $t$ for $L$-terms. If $\Theta$ is a term, formula, nominal form or squent then $F V(\Theta)$ denotes the set of all free variables occurring in $\Theta . \Theta$ is called closed iff $F V(\Theta)=\emptyset$.

\subsection{Assumptions}

(i) We choose a standard Gödel-numbering $\Theta \mapsto\ulcorner\Theta\urcorner$ of all syntactic objects (formulas, sequents, finite derivations, etc.) occurring in the paper. A set $\mathfrak{X}$ of syntactic objects is called primitive recursive if the set $\{\ulcorner\Theta\urcorner ; \Theta \in \mathfrak{X}\}$ has this property. An analogous agreement is made with respect to functions.

(ii) For each $n$-ary predicate symbol $p \in L$ we choose some fixed primitive recursive relation $\mathbf{p} \subseteq \mathbb{N}^{n}$. The set of all closed prime formulas which are true under this interpretation is denoted by $T R U E$, i.e.

$$
\begin{aligned}
\text { TRUE: }: & =\left\{p k_{1} \ldots k_{n}: p \in L \text { and }\left(k_{1}, \ldots, k_{n}\right) \in \mathbf{p}\right\} \\
& \cup\left\{\sim p k_{1} \ldots k_{n}: p \in L \text { and }\left(k_{1} \ldots k_{n}\right) \notin \mathbf{p}\right\} .
\end{aligned}
$$

We assume that $T R U E$ is primitive recursive.

(iii) We assume that $L$ contains some distinguished binary predicate symbol $p_{0}$ such that $\mathbf{p}_{0}$ is a wellordering of its field $\mathscr{A}$. In the following we always write $\prec$ for $\mathbf{p}_{0}$. Moreover we assume that there are primitive recursive functions $\oplus: \mathbb{N}^{2} \rightarrow \mathbb{N}$, 
$\exp : \mathbb{N} \rightarrow \mathbb{N}$ satisfying the following conditions:

$(\prec 1) \exp (0)=1$; and 0,1 are the first two elements of $(\mathscr{A}, \prec)$

$(<2) \forall a, b \in \mathscr{A}(a \oplus b \in \mathscr{A} \& \exp (a) \in \mathscr{A} \& a \oplus 0=0 \oplus a=0)$

(く3) $\forall a, b, c \in \mathscr{A}(b<a \Rightarrow c \oplus b \prec c \oplus a \& \exp (b)<\exp (a))$

$(\prec 4) \forall a, b, c \in \mathscr{A}(b, c<\exp (a) \Rightarrow b \oplus c \prec \exp (a))$.

\subsection{Abbreviations}

$m \nless n: \Leftrightarrow$ not $m<n$

$s<t: \equiv p_{0} s t$

$A \rightarrow B: \equiv \neg A \vee B$.

$\operatorname{Prog}_{\prec}(F): \equiv \forall x(\forall y(y \prec x \rightarrow F(y)) \rightarrow F(x))$.

$\Gamma, A:=\Gamma \cup A:=\Gamma \cup\{A\} ; \Gamma \backslash A:=\Gamma \backslash\{A\}$.

$F O R:=$ set of all $L$-formulas

$S E Q:=$ set of all sequents.

X-FOR: $=\left\{A \in F O R: A\right.$ is of shape $A_{0} \wedge A_{1}$ or $\left.\forall x F(x)\right\}$.

W-FOR: $=\left\{A \in F O R: A\right.$ is of shape $A_{0} \vee A_{1}$ or $\left.\exists x F(x)\right\}=\{\neg B: B \in M-F O R\}$.

For $A \in M-F O R \cup W-F O R$ we set

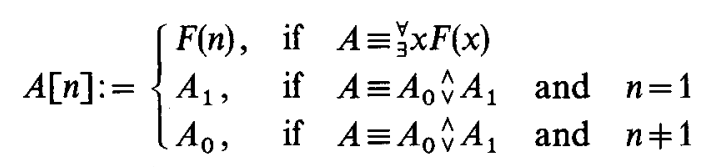

$\mathrm{W}_{0}-F O R:=\mathrm{W}-F O R \cup\{\neg A: A \in T R U E\}$

$\mathbb{N}^{<\omega}:=\left\{\left\langle n_{0}, \ldots, n_{k-1}\right\rangle: k, n_{0}, \ldots, n_{k-1} \in \mathbb{N}\right\}$;

$\left\langle m_{0}, \ldots, m_{\ell-1}\right\rangle *\left\langle n_{0}, \ldots, n_{k-1}\right\rangle:=\left\langle m_{0}, \ldots, m_{\ell-1}, n_{0}, \ldots, n_{k-1}\right\rangle$.

\langle\rangle$:=$ empty sequence.

$\alpha-1:=\left\{\begin{array}{lll}\alpha-1, & \text { if } & 0<\alpha<\omega \\ \alpha, & \text { if } & \alpha \in\{0, \omega\}\end{array}\right.$.

1.4 The formal system $Z+T I_{\prec \uparrow}$

$Z+T I_{\prec i}$ is the extension of classical arithmetic $Z$ (formulated in $L$ ) by the axioms of transfinite induction along every proper initial segment of $(\mathscr{A}, \prec)$.

The axioms of $Z+T I_{\prec i}$ (in sequent form) are:

(i) Basic axioms:

a) Logical axioms: $\{\neg A, A\}$, for every prime formula $A$.

b) Arithmetical axioms: Peano-axioms for 0 and', defining axioms for primitive recursive relations (in sequent form). [It is not necessary to display these axioms in detail; we only need to know that they are closed under substitution of $L$-terms, and that $\Gamma \cap T R U E \neq \emptyset$ for each closed arithmetical axiom $\Gamma$.]

(ii) Complete Induction: $\left\{\neg F(0), \neg \forall x\left(F(x) \rightarrow F\left(x^{\prime}\right)\right), \forall x F(x)\right\}$, for each $F$.

(iii) Transfinite Induction: $\left\{\neg \operatorname{Prog}_{\prec}(F), \forall x<m F(x)\right\}$, for each $F$ and each $m \in \mathscr{A}$.

The inference rules of $Z+T I_{\prec \uparrow}$ are:
$(\wedge) \frac{\Gamma, A_{0} \quad \Gamma, A_{1}}{\Gamma, A_{0} \wedge A_{1}}$
( $\forall) \frac{\Gamma, F(v)}{\Gamma, \forall x F(x)}$ with $v \notin F V(\Gamma, F)$,
( v) $\frac{\Gamma, A_{i}}{\Gamma, A_{0} \vee A_{1}}$,
(ヨ) $\frac{\Gamma, F(t)}{\Gamma, \exists x F(x)}$,
(Cut) $\frac{\Gamma, A \quad \Gamma, \neg A}{\Gamma}$. 
Usually one defines the derivations of a system like $Z+T I_{<1}$ as finite trees of sequents built up from axioms by the inference rules $(\wedge),(\vee),(\forall),(\exists),(\mathrm{Cut})$. But for the purpose of this paper we need a somewhat sharper notion of derivation, where at each node $\sigma$ of the tree also some special instance $r$ of the above rules is specified by which the sequent $\Gamma$ at node $\sigma$ can be derived from its premises $\ldots \Gamma_{n} \ldots$. Moreover we require that every free varible $v$ occurring in some premise of an inference $\mathbf{r}$ has to occur also in the conclusion, except $\mathbf{r}$ is an instance of $(\forall)$ and $v$ is its eigenvariable. Further we deviate a little bit from common practice in so far as we consider $\frac{\ldots \Gamma_{n} \ldots(n<\tau)}{\Gamma}$ as shorthand for "If, for each $n<\tau, d_{n}$ is a derivation with endsequent $\operatorname{End}\left(d_{n}\right) \cong \Gamma_{n}$, then $d:=\left\{\begin{array}{c}\ldots d_{n} \ldots \\ \bigvee / \\ \Gamma\end{array}\right.$ is a derivation with $\operatorname{End}(d)=\Gamma . "$ while usually one requires $\operatorname{End}\left(d_{n}\right)=\Gamma_{n}$.

Now we are going to give the "official" definition of the set $Z_{<\uparrow}$ of all $\left(Z+T I_{<\}}\right)$-derivations. The elements of $Z_{<}$will be finite trees of pairs $(\mathbf{r}, \Gamma)$ represented in linear notation, i.e. every $d \in Z_{\prec}$ is of the form $\left\langle\mathbf{r}, \Gamma,\left(d_{i}\right)_{i<\tau}\right\rangle$ with $E n d(d):=\Gamma \in S E Q, \tau \leqq 2$ and $d_{i} \in Z_{\prec}$. When writing down the defining rules for $Z_{\prec}$ we use the following abbreviation:

$$
d \Vdash \Gamma: \Leftrightarrow d \in Z_{\prec} \& \operatorname{End}(d) \subseteq \Gamma .
$$

\subsubsection{Inductive definition of the set $\boldsymbol{Z}_{<}$}

(Z.1) For every basic Axiom $\Gamma:\langle A x, \Gamma, \emptyset\rangle \in Z_{<}$

(Z.2) For each nominal form $F$ : $\left\langle(\right.$ Ind, $F), \quad\left\{\neg F(0), \quad \neg \forall x\left(F(x) \rightarrow F\left(x^{\prime}\right)\right)\right.$, $\forall x F(x)\}, \emptyset>\in Z_{\prec}$

(Z.3) For each nominal form $F$ and $m \in \mathscr{A}:\left\langle(\mathrm{TI}, F, m), \quad\left\{\neg \operatorname{Prog}_{\prec}(F)\right.\right.$, $\forall x<m F(x)\}, \emptyset\rangle \in Z_{\prec}$

(Z.4) $A_{0} \wedge A_{1} \in \Gamma \& d_{i} \mid \mapsto \Gamma \cup A_{i}(i=0,1) \Rightarrow\left\langle\left(\mathbb{A}, A_{0} \wedge A_{1}\right), \Gamma,\left(d_{0}, d_{1}\right)\right\rangle \in Z_{\prec}$

(Z.5) $A_{0} \vee A_{1} \in \Gamma \& d_{0} \Vdash \Gamma \cup A_{k}(k=0$ or 1$) \Rightarrow\left\langle\left(W_{k}, A_{0} \vee A_{1}\right), \Gamma, d_{0}\right\rangle \in Z_{\prec}$

(Z.6) $\forall x F(x) \in \Gamma \& d_{0} \Vdash \Gamma \cup F(v)$ with $v \notin F V(\Gamma) \Rightarrow\left\langle(v, \forall x F(x)), \Gamma, d_{0}\right\rangle \in Z_{\prec}$

(Z.7) $\exists x F(x) \in \Gamma \& d_{0} \| \Gamma \cup F(t)$ with $F V(t) \cong F V(\Gamma) \Rightarrow\left\langle\left(W_{t}, \exists x F(x)\right), \Gamma, d_{0}\right\rangle \in Z_{\prec}$

(Z.8) $d_{0} \Vdash \Gamma \cup A \& d_{1} \| \Gamma \cup \neg A$ with $F V(A) \subseteq F V(\Gamma) \Rightarrow\left\langle(\right.$ Cut,$\left.A), \Gamma,\left(d_{0}, d_{1}\right)\right\rangle \in Z_{\prec}$

1.4.2 Definition of the cut-rank $\operatorname{deg}(d) \in \mathbb{N}$ for $d \in Z_{\prec}$. Let $d=\left\langle\mathbf{r}, \Gamma,\left(d_{i}\right)_{i<\tau}\right\rangle \in Z_{\prec}$.

where

$$
\operatorname{deg}(d):=\max \left(\left\{\operatorname{deg}\left(d_{i}\right): i<\tau\right\} \cup\left\{\ell_{\mathbf{C}}(\mathbf{r})\right\}\right),
$$

$$
\ell_{C}(\mathbf{r}):=\left\{\begin{array}{ll}
\ell(A)+1, & \text { if } \mathbf{r}=(\text { Cut }, A) \\
0, & \text { otherwise }
\end{array} .\right.
$$

\section{The system $Z^{\text {o }}$ and the cut-elimination operator $\mathscr{E}$}

Combining work of Schütte [13], Tait [17], and Minc [10] we shall now describe the system $Z^{\infty}$ of wellfounded infinitary derivations (of $\omega$-arithmetic), and the operator $\mathscr{E}$ transforming each $Z^{\infty}$-derivation with finite cut-rank $m$ into a derivation of the same sequent but with cut-rank $\leqq m-1$. Informally $Z^{\infty}$ is defined as the set of all wellfounded trees (derivations) generated from initial sequents $\Gamma$ 
with $\Gamma \cap T R U E \neq \emptyset$ by the rules $(\wedge),(\vee),(\mathrm{Cut}),(\exists)[$ with witness $t \in \mathbb{N}]$ and

$$
(\forall)^{\infty} \frac{\ldots \Gamma, F(n) \ldots(n \in \mathbb{N})}{\Gamma, \forall x F(x)}\left(\omega \text {-rule), (Rep) } \frac{\Gamma}{\Gamma}\right. \text { (repetition-rule). }
$$

But as in 1.4 we will consider a modified notion of derivation, where to each node is assigned a pair $(\mathbf{r}, \Gamma)$ with $\Gamma \in S E Q$ and $\mathbf{r}$ an expression indicating some special instance of the above inference rules. The set of all these expressions will be called RULE. Then the inference rules can be written as local correctness conditions $(L C .1), \ldots,(L C .5)$ between a pair $(\mathbf{r}, \Gamma) \in R U L E \times S E Q$ and a family $\left(\Gamma_{n}\right)_{n \in I}$ of sequents (the premises of $\Gamma$ ); and $Z^{\infty}$ is defined as the set of all wellfounded ( $R U L E$ $\times S E Q)$-trees which are locally correct with respect to $(L C 1), \ldots,(L C .5)$.

\subsection{Definition}

$$
\begin{aligned}
R U L E:= & \{\mathrm{Ax}\} \cup\{(\mathbb{M}, A): A \in \mathbb{X}-F O R\} \cup\left\{\left(W_{k}, A\right): k \in \mathbb{N}, A \in \mathbb{W}-F O R\right\} \\
& \cup\left\{\operatorname{Rep}_{n}: n \in \mathbb{N}\right\} \cup\{(\text { Cut }, A): A \in F O R\} .
\end{aligned}
$$

For $\mathbf{r} \in R U L E$ we use the following abbreviations

$(\mathbf{r}, \Gamma) \in \mathrm{Ax}: \Leftrightarrow \mathbf{r}=\mathrm{Ax}$,

$\mathrm{r} \in \mathrm{Cut}: \Leftrightarrow \mathbb{I} A[\mathrm{r}=($ Cut,$A)], \mathrm{r} \in \operatorname{Rep}: \Leftrightarrow \mathbb{I} n\left[\mathrm{r}=\operatorname{Rep}_{n}\right]$,

$\ell_{C}(\mathbf{r}):= \begin{cases}\ell_{c}(A)+1, & \text { if } \mathbf{r}=(\text { Cut }, A) \\ 0, & \text { otherwise }\end{cases}$

2.2 Definition. $L C\left((\mathbf{r}, \Gamma),\left(\Gamma_{n}\right)_{n \in \mathbb{N}}\right)$ abbreviates the conjunction of $(L C .1), \ldots,(L C .5)$ below:

(LC.1) $\mathbf{r}=\mathrm{Ax} \Rightarrow \Gamma \cap T R U E \neq \emptyset$

$(L C .2) \mathbf{r}=(\mathbb{M}, A) \Rightarrow A \in \Gamma \& \forall n\left(\Gamma_{n} \subseteq \Gamma \cup A[n]\right)$

(LC.3) $\mathbf{r}=\left(\mathrm{W}_{k}, A\right) \Rightarrow A \in \Gamma \& \Gamma_{0} \subseteq \Gamma \cup A[k]$

(LC.4) $\mathrm{r}=$ (Cut, $A) \Rightarrow \Gamma_{0} \subseteq \Gamma \cup A \& \Gamma_{1} \leqq \Gamma \cup \neg A$

(LC.5) $\mathrm{r}=\operatorname{Rep}_{n} \Rightarrow \Gamma_{n} \subseteq \Gamma$.

\subsection{Definitions}

a) Let $T R E E$ be the set of all functions $\varphi: \mathbb{N}^{<\omega} \rightarrow R U L E \times S E Q$ satisfying the condition

$$
\forall \sigma \in \mathbb{N}^{<\omega} \mathbb{Z} n \in \mathbb{N}[\varphi(\sigma) \in \mathrm{Ax} \Rightarrow \varphi(\sigma *\langle n\rangle)=\varphi(\sigma)] .
$$

b) $\mathbf{0}_{(r, \Gamma)}$ denotes the constant tree $\varphi$ with $\varphi(\sigma)=(\mathbf{r}, \Gamma)$ for all $\sigma \in \mathbb{N}^{<\omega}$.

c) For $\varphi \in T R E E$ we define:

(i) $\varphi^{0}(\sigma):=\mathbf{r}, \varphi^{1}(\sigma):=\Gamma$, with $(\mathbf{r}, \Gamma):=\varphi(\sigma)$.

(ii) Rule $(\varphi):=\varphi^{0}(\langle\rangle), \operatorname{End}(\varphi):=\varphi^{1}(\langle\rangle)$.

(iii) $\varphi[n]:=\lambda \sigma \cdot \varphi(\langle n\rangle * \sigma) \in T R E E$ (the $n$-th immediate subtree of $\varphi$ ). $\varphi \llbracket \tau \rrbracket:=\lambda \sigma . \varphi(\tau * \sigma) \in T R E E$ (the subtree of $\varphi$ determined by $\tau$ ).

(iv) $\varphi$ is wellfounded: $\Leftrightarrow \forall\left(n_{i}\right)_{i \in \mathrm{N}}$ 四 $k\left(\varphi\left(\left\langle n_{0}, \ldots, n_{k-1}\right\rangle\right) \in \mathrm{Ax}\right)$.

(v) $\varphi$ is locally correct $: \Leftrightarrow \forall \sigma \in \mathbb{N}^{<\omega} L C\left(\varphi(\sigma),\left(\varphi^{1}(\sigma *\langle n\rangle)\right)_{n \in \mathbb{N}}\right)$. Notation. We use $\varphi$ as syntactical variable for elements of TREE.

\subsection{Remark}

a) $\varphi \in T R E E \& \varphi(\langle\rangle)=(\operatorname{Ax}, \Gamma) \Rightarrow \varphi=\mathbf{0}_{(\mathrm{Ax}, \Gamma)}$.

b) $\varphi \in T R E E \Rightarrow\left[\forall n(\varphi[n]=\varphi) \Leftrightarrow \varphi=\mathbf{0}_{\varphi(\langle\rangle)}\right]$.

\subsection{Definition}

$W T:=\{\varphi \in T R E E: \varphi$ wellfounded $\}$

$Z^{\infty}:=\{\varphi \in W T: \varphi$ locally correct $\}$. 
2.6 Proposition (Inductive definition of $W T$ and $Z^{\infty}$ )

a) $W T$ is the least subset of TREE satisfying

(WT.0) $\mathbf{0}_{(\mathrm{Ax}, \Gamma)} \in W T$, for each $\Gamma \in S E Q$.

(WT.1) Rule $(\varphi) \neq A x \& \forall n \in \mathbb{N}(\varphi[n] \in W T) \Rightarrow \varphi \in W T$.

b) $Z^{\infty}$ is the least subset of TREE satisfying

$\left(Z^{\infty} .0\right) \mathbf{0}_{(\mathrm{Ax}, \Gamma)} \in Z^{\infty}$, for each $\Gamma$ with $\Gamma \cap T R U E \neq \emptyset$,

$\left(Z^{\infty} .1\right)$ Rule $(\varphi) \neq A \times \& \forall n \in \mathbb{N}\left(\varphi[n] \in Z^{\infty}\right) \& L C\left(\varphi(\langle\rangle),(\operatorname{End}(\varphi[n]))_{n \in \mathbb{N}}\right)$ $\Rightarrow \varphi \in Z^{\infty}$.

2.7 Definition of $\operatorname{deg}(\varphi) \leqq \omega$ for $\varphi \in T R E E$

$\operatorname{deg}(\varphi):=\sup \left\{\ell_{C}\left(\varphi^{0}(\sigma)\right): \sigma \in \mathbb{N}^{<\omega}\right\}\left(=\sup \left\{\ell(A)+1: \exists \operatorname{Il} \sigma \in \mathbb{N}^{<\omega}\left(\varphi^{0}(\sigma)=(\right.\right.\right.$ Cut,$\left.\left.\left.A)\right)\right\}\right)$.

2.8 Definition of $\|\varphi\| \in O n$ for $\varphi \in W T$

$$
\|\varphi\|:=\left\{\begin{array}{ll}
0, & \text { if } \operatorname{Rule}(\varphi)=\mathrm{Ax} \\
\sup \{\|\varphi[n]\|+1: n \in \mathbb{N}\}, & \text { otherwise }
\end{array} .\right.
$$

Now we are going to define the cut-elimination operator $\mathscr{E}: W T \rightarrow W T$ which transforms every $\varphi \in Z^{\infty}$ into a derivation $\mathscr{E}(\varphi) \in Z^{\infty}$ with $\operatorname{End}(\mathscr{E}(\varphi))=\operatorname{End}(\varphi)$ and $\operatorname{deg}(\mathscr{E}(\varphi)) \leqq \operatorname{deg}(\varphi)-1$. For the definition of $\mathscr{E}$ we need two families of auxiliary operators, namely the inversion operators $\mathscr{I}_{k, A}(k \in \mathbb{N}, A \in M-F O R)$ and the reduction operators $\mathscr{R}_{C}\left(C \in W_{0}-F O R\right)$. The operator $\mathscr{I}_{k, A}$ replaces in $\varphi$ every sequent $\Gamma$ by $(\Gamma \backslash A) \cup A[k]$, and every occurrence of $(\mathcal{M}, A)$ by $\operatorname{Rep}_{k}$. The effect of $\mathscr{R}_{C}$ is to reduce a cut with cutformula $C$ to cuts with cutformulas $C[k]$; namely if $\psi$, $\varphi \in Z^{\infty}$ are derivations with $\operatorname{End}(\psi) \subseteq \Gamma \cup \neg C, \operatorname{End}(\varphi) \subseteq \Gamma \cup C$ and $\max \{\operatorname{deg}(\psi)$, $\operatorname{deg}(\varphi)\} \leqq \ell(C)$ then $\tilde{\varphi}:=\mathscr{R}_{C}(\psi, \varphi) \in Z^{\infty}$ is a derivation with $\operatorname{End}(\tilde{\varphi}) \subseteq \Gamma$ and $\operatorname{deg}(\tilde{\varphi})$ $\leqq \ell(C)$. - The definitions of $\mathscr{I}_{k, A}(\varphi), \mathscr{R}_{C}(\psi, \varphi), \mathscr{E}(\varphi)$ proceed by transfinite recursion on $\|\varphi\|$ using the fact that every $\varphi \in T R E E$ is uniquely determined by the data $\operatorname{End}(\varphi), \operatorname{Rule}(\varphi),(\varphi[n])_{n \in \mathbb{N}}$.

2.9 Definition of the inversion operator $\mathscr{I}_{k, A}: W T \rightarrow W T$ for $A \in M-F O R, k \in \mathbb{N}$

(i) $\operatorname{End}\left(\mathscr{I}_{k, A}(\varphi)\right):=(\operatorname{End}(\varphi) \backslash A) \cup A[k]$

(ii) Rule $\left(\mathscr{I}_{k, A}(\varphi)\right):= \begin{cases}\operatorname{Rep}_{k}, & \text { if } \operatorname{Rule}(\varphi)=(\mathbb{M}, A) \\ \operatorname{Rule}(\varphi), & \text { otherwise }\end{cases}$

(iii) $\mathscr{I}_{k, A}(\varphi)[n]:=\mathscr{I}_{k, A}(\varphi[n])$.

Explanation. Let $\mathscr{I}$ abbreviate $\mathscr{I}_{k, \boldsymbol{A}}$. If $\operatorname{Rule}(\varphi) \neq \mathrm{Ax}$ then in defining $\mathscr{I}(\varphi)[n]$ we can refer to the previously defined $\mathscr{I}(\varphi[n])$, which is not possible when $\operatorname{Rule}(\varphi)$ $=\mathrm{Ax}$. In that case we have $\varphi=\mathbf{0}_{(\mathrm{Ax}, \Gamma)}$, and (iii) becomes $\mathscr{I}(\varphi)[n]:=\mathscr{I}(\varphi)$ which is circular. But according to $2.4 \mathrm{~b}$ ) we consider this as an abbreviation for $\mathscr{I}(\varphi):=\mathbf{0}_{\left(\mathbf{r}, \Gamma^{\prime}\right)}$ with $\Gamma^{\prime}:=\operatorname{End}(\mathscr{I}(\varphi)), \mathbf{r}:=\operatorname{Rule}(\mathscr{I}(\varphi))$ as defined under (i) and (ii). Moreover in that case by (ii) we have $\mathbf{r}=\mathrm{Ax}$ and thus $\mathscr{I}(\varphi)=\mathbf{0}_{\left(\mathrm{Ax}, \Gamma^{\prime}\right)} \in W T$. An analogues remark applies to the definitions of $\mathscr{R}_{C}$ and $\mathscr{E}$ below.

2.10 Definition of $\mathscr{R}_{C}: W T \times W T \rightarrow W T$ for $C \in W_{0}-F O R$

$\operatorname{End}\left(\mathscr{R}_{C}(\psi, \varphi)\right):=(\operatorname{End}(\psi) \backslash \neg C) \cup(\operatorname{End}(\varphi) \backslash C)$

$\operatorname{Rule}\left(\mathscr{R}_{C}(\psi, \varphi)\right):= \begin{cases}(\mathrm{Cut}, C[k]), & \text { if } \operatorname{Rule}(\varphi)=\left(\mathrm{W}_{k}, C\right) \\ \operatorname{Rule}(\varphi), & \text { otherwise }\end{cases}$

$\mathscr{R}_{C}(\psi, \varphi)[n]:= \begin{cases}\mathscr{I}_{k, \neg C}(\psi), & \text { if } \operatorname{Rule}(\varphi)=\left(\mathrm{W}_{k}, C\right) \text { and } n=1 \\ \mathscr{R}_{C}(\psi, \varphi[n]), & \text { otherwise }\end{cases}$ 
2.11 Definition of $\mathscr{E}: W T \rightarrow W T$

$\operatorname{End}(\mathscr{E}(\varphi)):=\operatorname{End}(\varphi)$.

$\operatorname{Rule}(\mathscr{E}(\varphi)):= \begin{cases}\operatorname{Rep}_{0}, & \text { if } \operatorname{Rule}(\varphi) \in \text { Cut } \\ \operatorname{Rule}(\varphi), & \text { otherwise }\end{cases}$

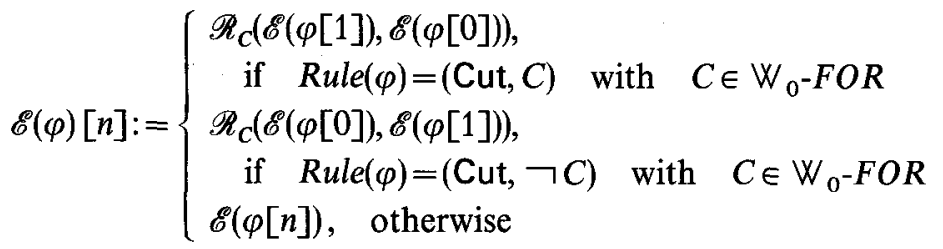

2.12 Proposition. For all $\psi, \varphi \in Z^{\infty}, A \in \mathbb{X}_{-F O R,} \in \in \mathbb{N}, C \in \mathbb{W}_{0}-F O R$ we have:

a) $\mathscr{I}_{k, A}(\varphi) \in Z^{\infty}, \operatorname{deg}\left(\mathscr{I}_{k, A}(\varphi)\right) \leqq \operatorname{deg}(\varphi),\left\|\mathscr{I}_{k, A}(\varphi)\right\| \leqq\|\varphi\|$

b) $\mathscr{R}_{C}(\psi, \varphi) \in Z^{\infty}, \operatorname{deg}\left(\mathscr{R}_{C}(\psi, \varphi)\right) \leqq \max \{\ell(C), \operatorname{deg}(\psi), \operatorname{deg}(\varphi)\}$, $\left\|\mathscr{R}_{\mathbf{C}}(\psi, \varphi)\right\| \leqq\|\psi\|+\|\varphi\|$

c) $\mathscr{E}(\varphi) \in Z^{\infty}, \operatorname{deg}(\mathscr{E}(\varphi)) \leqq \operatorname{deg}(\varphi)-1,\|\mathscr{E}(\varphi)\| \leqq \omega^{\|\varphi\|}$.

Proof by induction on $\|\varphi\|$.

2.13 Definition of $Z_{\prec}^{0} . Z_{\prec}^{0}:=\left\{d \in Z_{\prec}: \operatorname{End}(d)\right.$ closed $\}$.

2.14 Proposition. There is a canonical embedding $Z_{\prec}^{0} \rightarrow Z^{\infty}, d \mapsto d^{\infty}$ such that $\operatorname{End}\left(d^{\infty}\right)=\operatorname{End}(d)$ and $\operatorname{deg}\left(d^{\infty}\right)=\operatorname{deg}(d) . d^{\infty}$ is obtained from d essentially by replacing in $d$ every axiom of complete or transfinite induction by its (cutfree) $\omega$-derivation.

\section{The notation system $Z_{<}^{*}$}

Our goal is to show that every provably recursive function of $Z+T I_{\prec \uparrow}$ can be represented as $\mathrm{f}(n)=\mathrm{g}(n, \min \{k: \mathrm{o}(n, k+1) \nless \mathrm{o}(n, k)\})$ with prim. rec. function $\mathrm{g}, \mathrm{o}$. Of course it suffices to show that for each $p \in L$ with $Z+T I_{\prec \uparrow} \vdash \forall x \exists y p x y$ the Skolem-function $\mathrm{f}_{p}(n):=\min \{m: \mathbf{p}(n, m)\}$ has such a representation. We want to do this by methods formalizable in $P R A+P R W O(\prec)$, i.e. primitive recursive arithmetic together with the axiom that there are no primitive recursive infinite descending sequences in $(\mathscr{A}, \prec)$. This will be achieved as follows: we introduce a primitive recursive system $Z_{\prec}^{*}$ of (finite) notations for all those $\varphi \in Z^{\infty}$ which can be obtained via cut-elimination from derivations $d^{\infty}\left(d \in Z_{\prec}^{0}\right)$. Then we define primitive recursive functions $\mathfrak{e}: Z_{\prec}^{*} \rightarrow S E Q, r: Z_{\prec}^{*} \rightarrow R U L E, \mathfrak{s}: \mathbb{N} \times Z_{\prec}^{*} \rightarrow Z_{\prec}^{*}, \mathfrak{D}: Z_{\prec}^{*}$ $\rightarrow \mathbb{N}$ such that, for all $h \in Z_{\prec}^{*}, n \in \mathbb{N}, \mathfrak{e}(h)=\operatorname{End}(\mathbf{v}(h)), \mathfrak{r}(h)=\operatorname{Rule}(\mathbf{v}(h)), \mathbf{v}(\mathfrak{s}(n, h))$ $=\mathbf{v}(h)[n], \operatorname{deg}(\mathbf{v}(h)) \leqq \mathfrak{D}(h)$, where $\mathbf{v}(h)$ is the value of $h$, i.e. the $Z^{\infty}$-derivation denoted by $h$.

Notation. $\mathfrak{s}_{n} h: \equiv \mathfrak{s}_{n}(h): \equiv \mathfrak{s}(n, h)$.

We also define a prim. rec. function $\mathfrak{o}: Z_{\prec}^{*} \rightarrow \mathscr{A}$ such that $[\mathfrak{r}(h)$ $\left.\neq \mathrm{Ax} \Rightarrow \mathfrak{v}\left(\mathfrak{s}_{n} h\right) \prec \mathfrak{v}(h)\right]$, for all $h \in Z_{\prec}^{*}, n \in \mathbb{N}$. (Thus $\mathfrak{o}(h)$ corresponds to $\left.\|\mathbf{v}(h)\|\right)$.

Now assume that $Z+T I_{\prec \uparrow} \vdash A$, where $A \equiv \forall x \exists y p x y$. Then there exists some $d \in Z_{\prec}^{0}$ with $\operatorname{End}(d) \subseteq\{A\}$ and $v:=\operatorname{deg}(d)<\omega$.

Let

$$
\begin{aligned}
& \varphi_{n, 0}:=\mathscr{I}_{n, A^{E}} \mathscr{E}^{(v)}\left(d^{\infty}\right), \\
& \varphi_{n, k+1}:= \begin{cases}\varphi_{n, k}[i], & \text { if } \operatorname{Rule}\left(\varphi_{n, k}\right)=\operatorname{Rep}_{i}, \quad \Gamma_{n, k}:=\operatorname{End}\left(\varphi_{n, k}\right) . \\
\varphi_{n, k}[0], & \text { otherwise, }\end{cases}
\end{aligned}
$$


Then by induction on $k$ we get: $\Gamma_{n, k} \subseteq\{\exists y p n y, p n 0, p n 1, \ldots\}$, and thus

$$
\operatorname{Rule}\left(\varphi_{n, k}\right)=\mathrm{Ax} \Rightarrow \exists I m \leqq \mathrm{U}\left(\Gamma_{n, k}\right) \mathrm{p}(n, m),
$$

with

$$
\mathrm{u}(\Gamma):=\sup \{m: m \text { occurs in } \Gamma\} .
$$

Using $\mathfrak{r}, \mathfrak{s}$ we can define a prim. rec. function $s: \mathbb{N}^{2} \rightarrow Z_{\prec}^{*}$ such that $\mathrm{v}(s(n, k))$ $=\varphi_{n, k}$.

Then $\mathbf{s}(n, k+1) \in\left\{\mathfrak{s}_{i} \mathbf{s}(n, k): i \in \mathbb{N}\right\}$ and therefore

$$
\mathfrak{r}(\mathbf{s}(n, k)) \neq \mathrm{Ax} \Rightarrow \mathrm{o}(n, k+1) \prec \mathrm{o}(n, k),
$$

with

$$
\mathrm{o}(n, k):=\mathrm{o}(\mathrm{s}(n, k)) .
$$

Using $\mathrm{r}(\mathrm{s}(n, k))=\operatorname{Rule}\left(\varphi_{n, k}\right), \mathrm{e}(\mathrm{s}(n, k))=\Gamma_{n, k}$ and (1), (2) we obtain

$$
\mathrm{o}(n, k+1) \nless \mathrm{o}(n, k) \Rightarrow \mathrm{f}_{p}(n)=\mathrm{g}(n, k),
$$

with

$$
\mathrm{g}(n, k):=\min \{m \leqq \mathrm{u}(\mathrm{e}(\mathrm{s}(n, k))): \mathbf{p}(n, m)\} .
$$

Since by $P R W O(<)$ we have $\forall n \mathbb{I I} k(\mathrm{o}(n, k+1) \nless \mathrm{o}(n, k))$, this yields

$$
\mathrm{f}_{p}(n)=\mathrm{g}(n, \min \{k: \mathrm{o}(n, k+1) \nless \mathrm{o}(n, k)\}) .
$$

Remark. The above argument cannot directly be formalized in $P R A$, since there we cannot define the interpretation $\mathrm{v}: Z_{\prec}^{*} \rightarrow Z^{\infty}$. But as we will see below this is really not necessary. It will suffice to prove in $P R A$ the $\Pi_{1}^{0}$-statement

$$
\begin{aligned}
& \forall h \in Z_{<}^{*}\left[L C\left((\mathfrak{r}(h), \mathfrak{e}(h)),\left(\mathfrak{e}\left(\mathfrak{s}_{n} h\right)\right)_{n \in \mathbb{N}}\right) \& \ell_{C}(\mathfrak{r}(h)) \leqq \mathrm{d}(h) \& \forall n\left(\mathfrak{d}\left(\mathfrak{s}_{n}(h) \leqq \mathfrak{d}(h)\right)\right.\right. \\
& \left.\&\left[\mathrm{r}(h) \neq \mathrm{Ax} \Rightarrow \forall n\left(\mathrm{o}\left(\mathfrak{s}_{n} h\right) \prec \mathrm{o}(h)\right)\right]\right] .
\end{aligned}
$$

3.1 Definition of the set $Z_{\prec}^{*}$. Since we want that $Z_{\prec}^{*}$ contains notations just for all $\varphi \in Z^{\infty}$ obtainable from derivations $d^{\infty}\left(d \in Z_{\prec}^{0}\right)$ by applications of the operators $\mathscr{I}_{k, A}, \mathscr{R}_{C}, \mathscr{E}$, we now introduce function symbols $I_{k, A}, R_{C}, E\left(k \in \mathbb{N}, A \in \mathbb{A}_{-} F O R\right.$, $\left.C \in W_{0}-F O R\right)$ and define

$$
Z_{<}^{*}:=\left\{\begin{array}{l}
\text { the set of all (finite) terms } h \text { generated from } \\
\text { "constants" } d \in Z_{\prec}^{0} \text { by the function symbols } I_{k, A}, R_{C}, E .
\end{array}\right.
$$

3.2 Definition of the value $\mathrm{v}(h) \in Z^{\infty}$ for each $h \in Z_{\prec}^{*}$

(i) $\mathbf{v}(h):=h^{\infty}$, if $h \in Z_{<}^{0}$;

(ii) $\mathbf{v}\left(I_{k, A} h\right):=\mathscr{I}_{k, A}(\mathbf{v}(h)) ; \mathbf{v}\left(R_{C}\left(h_{0} h_{1}\right):=\mathscr{R}_{C}\left(\mathbf{v}\left(h_{0}\right), \mathbf{v}\left(h_{1}\right)\right) ; \mathbf{v}(E h):=\mathscr{E}(\mathbf{v}(h))\right.$.

3.3 Definition of the length $\ell^{*}(h) \in \mathbb{N}$ for each $h \in Z^{*}$

$$
\begin{gathered}
\ell^{*}(h):=0, \text { if } h \in Z_{<}^{0} ; \quad \ell^{*}(E h):=\ell^{*}\left(I_{k, A} h\right):=\ell^{*}(h)+1, \\
\ell^{*}\left(R_{C} h_{0} h_{1}\right):=\max \left\{\ell^{*}\left(h_{0}\right), \ell^{*}\left(h_{1}\right)\right\}+1 .
\end{gathered}
$$

Now we are going to define the above mentioned functions

$$
\begin{gathered}
\mathfrak{e}: Z_{\prec}^{*} \rightarrow S E Q, \quad \mathfrak{r}: Z_{\prec}^{*} \rightarrow R U L E, \quad \mathfrak{s}: \mathbb{N} \times Z_{\prec}^{*} \rightarrow Z_{\prec}^{*}, \\
\mathfrak{d}: Z_{\prec}^{*} \rightarrow \mathbb{N}, \quad \mathfrak{0}: Z_{\prec}^{*} \rightarrow \mathscr{A} .
\end{gathered}
$$


The definition proceeds by (primitive) recursion on $\ell^{*}(h)$. First we define e $(d)$, $\mathfrak{r}(d), \ldots$ for all $d \in Z_{<}^{0}$, and then we define $\mathrm{e}\left(I_{k, A} h_{1}\right), \mathfrak{e}\left(R_{C} h_{0} h_{1}\right), \mathfrak{e}\left(E h_{1}\right), \mathfrak{r}\left(I_{k, A} h_{1}\right), \ldots$ under the induction hypothesis that $\mathrm{e}\left(h_{i}\right), \mathfrak{r}\left(h_{i}\right), \ldots$ are already defined. The defining equations for $e, r, \mathfrak{s}$ in the induction step are obtained by a simple rewriting from the corresponding equations for $\mathscr{I}_{k, A}, \mathscr{R}_{C}, \mathscr{E}$ on pp. 283, 284. But of course both definitions are fundamentally different with respect to the underlying recursion principle.

3.4 Definition of $\mathrm{e}(d), \mathfrak{r}(d), \mathfrak{s}_{n}(d)$ for $d \in Z_{\prec}$. Let $d=\left\langle\mathrm{r}, \Gamma,\left(d_{i}\right)_{i<\imath}\right\rangle \in Z_{\prec}$. $\mathrm{e}(d):=\Gamma=\operatorname{End}(d)$

$$
\begin{gathered}
\mathfrak{r}(d):= \begin{cases}(\mathbb{A}, \forall x F(x)), & \text { if } \mathbf{r}=(v, \forall x F(x)) \text { or } \quad \text { (Ind, } F) \\
(\mathbb{A}, \forall x<m F(x)), & \text { if } \mathbf{r}=(\mathrm{TI}, F, m) \\
\mathbf{r}, & \text { otherwise }\end{cases} \\
\mathfrak{s}_{n}(d):= \begin{cases}d, & \text { if } \mathbf{r}=\mathrm{Ax} \\
c_{n}^{F}, & \text { if } \mathbf{r}=(\operatorname{Ind}, F) \\
c_{\mathrm{m}, \mathrm{n}}^{\mathrm{F}}, & \text { if } \mathbf{r}=(\mathrm{TI}, F, m) \\
d_{0}[v / n], & \text { if } \mathbf{r}=(v, \forall x F(x)) \\
d_{n}, & \text { if } \tau=2 \text { and } n<2 \\
d_{0}, & \text { otherwise }\end{cases}
\end{gathered}
$$

$c_{n}^{F}$ and $c_{m, n}^{F}$ are defined below. For each $n \in \mathbb{N}, d_{0}[v / n]$ denotes the result of substituting $n$ for every occurrence of $v$ in $d_{0}$ which is "linked" to the root of $d_{0}$.

Definition of $\kappa[A]$. For $A \in F O R$ let $\kappa[A] \in Z_{\prec}$ be the canonical cutfree derivation of $\{\neg A, A\}$.

Definition of $c_{n}^{F}$. Let $G: \equiv \neg \forall x\left(F(x) \rightarrow F\left(x^{\prime}\right)\right) \equiv \exists x\left(F(x) \wedge \neg F\left(x^{\prime}\right)\right)$.

with

$$
\begin{gathered}
c_{0}^{F}:=\kappa[F(0)] \\
c_{n+1}^{F}:=\left\langle\left(W_{n}, G\right), \Gamma,\left\langle\left(\mathbb{M}, F(n) \wedge \neg F\left(n^{\prime}\right)\right), \Gamma_{n},\left(c_{n}^{F}, \kappa\left[F\left(n^{\prime}\right)\right]\right)\right\rangle\right\rangle
\end{gathered}
$$

$$
\Gamma:=\left\{\neg F(0), G, F\left(n^{\prime}\right)\right\}, \quad \Gamma_{n}:=\left\{\neg F(0), F, F(n) \wedge \neg F\left(n^{\prime}\right), F\left(n^{\prime}\right)\right\} .
$$

To improve readability we repeat the definition of $c_{n+1}^{F}$ in familiar notation:

Definition of $c_{m, n}^{F}$

$$
c_{n+1}^{F}:=\left\{\begin{array}{cc}
c_{n}^{F} & \kappa\left[F\left(n^{\prime}\right)\right] \\
\frac{\neg F(0), G, F(n)-\neg F\left(n^{\prime}\right), F\left(n^{\prime}\right)}{\neg F(0), G, F(n) \wedge \neg F\left(n^{\prime}\right), F\left(n^{\prime}\right)} & (\wedge) \\
\left.\frac{\neg F(0), G, F\left(n^{\prime}\right)}{(}\right)
\end{array}\right.
$$

(i) $n \nless m: c_{m, n}^{F}:=\left\langle\left(W_{0}, n \prec m \rightarrow F(n)\right),\{n \prec m \rightarrow F(n)\},\langle A x,\{\neg n\langle m\}, \emptyset\rangle\rangle\right.$

(ii) $n \prec m$ : Let $G: \equiv \neg \operatorname{Prog}_{\prec}(F)$, i.e. $G \equiv \exists x(\forall y \prec x F(y) \wedge \neg F(x))$.

$$
\begin{aligned}
c_{m, n}^{F}:= & \left\langle\left(W_{1}, \neg n \prec m \vee F(n)\right), \Delta,\left\langle\left(W_{n}, G\right), \Delta^{\prime},\langle(\mathbb{M}, \forall y<n F(y)\right.\right. \\
& \left.\left.\left.\wedge \neg F(n)), \Delta^{\prime \prime},(e, \kappa[F(n)])\right\rangle\right\rangle\right\rangle
\end{aligned}
$$

with

$$
e:=\langle(T I, F, n),\{G, \forall y \prec n F(y)\}, \emptyset\rangle
$$


and

$$
\begin{gathered}
\Delta:=\{G, n<m \rightarrow F(n)\}, \quad \Delta^{\prime}:=\{G, F(n)\}, \\
\Delta^{\prime \prime}:=\{G, \forall y \prec n F(y) \wedge \neg F(n), F(n)\} .
\end{gathered}
$$

In familiar notation:

$$
c_{m, n}^{F}:=\left\{\begin{array}{cc}
\kappa[F(n)] \\
\frac{\frac{G, \forall y<n F(y)-\neg F(n), F(n)}{G, \forall y<n F(y) \wedge \neg F(n), F(n)}(\wedge)}{\frac{G, F(n)}{G, n<m \rightarrow F(n)}(\vee)}
\end{array}\right.
$$

3.5 Lemma. $d \in Z_{\prec}^{0} \Rightarrow \mathrm{r}(d) \in R U L E$ and $\mathfrak{s}_{n} d \in Z_{\prec}^{0}$.

Proof. Straightforward, using the fact that for $d=\left\langle\mathbf{r}, \Gamma,\left(d_{i}\right)_{i<\tau}\right\rangle \in Z_{\prec}^{0}$ one has $F V\left(\operatorname{End}\left(d_{i}\right)\right) \subseteq\left\{\begin{array}{ll}\{v\}, & \text { if } \mathbf{r}=(v, \forall x F(x)) \\ \emptyset, & \text { otherwise }\end{array}\right.$ and $\left[\mathbf{r}=\left(W_{t}, \exists x F(x)\right) \Rightarrow t \in \mathbb{N}\right]$.

3.6 Definition of $\mathfrak{D}(d) \in \mathbb{N}$ and $\mathfrak{p}(d) \in \mathscr{A}$ for $d \in Z_{\prec}$. Let $d=\left\langle\mathbf{r}, \Gamma,\left(d_{i}\right)_{i<\tau}\right\rangle$.

$$
\mathfrak{o}(d):= \begin{cases}\exp (1), & \text { if } \mathbf{r}=(\operatorname{lnd}, F) \\ \prec-\max \{\exp (m), 1 \oplus 1\}, & \text { if } \mathbf{r}=(\mathrm{TI}, F, m) \\ \prec-\sup \left\{\mathfrak{o}\left(d_{i}\right) \oplus 1: i<\tau\right\}, & \text { otherwise }\end{cases}
$$

3.7 Definition of $\mathfrak{e}(h), \mathfrak{r}(h), s_{n}(h), \mathfrak{d}(h), \mathfrak{o}(h)$ for $h \in Z_{\prec}^{*} \backslash Z_{\prec}^{0}$

$\mathfrak{e}\left(I_{k, A} h\right):=(\mathfrak{e}(h) \backslash A) \cup A[k], \mathfrak{e}\left(R_{C} h_{0} h_{1}\right):=\left(\mathfrak{e}\left(h_{0}\right) \backslash \neg C\right) \cup\left(\mathfrak{e}\left(h_{1}\right) \backslash C\right), \mathfrak{e}(E h):=\mathfrak{e}(h)$.

$\mathfrak{r}\left(I_{k, A} h\right):= \begin{cases}\operatorname{Rep}_{k}, & \text { if } \mathfrak{r}(h)=(M, A) \\ \mathfrak{r}(h), & \text { otherwise. }\end{cases}$

$\mathfrak{r}\left(R_{C} h_{0} h_{1}\right):= \begin{cases}(\text { Cut, } C[k]), & \text { if } \mathfrak{r}\left(h_{1}\right)=\left(W_{k}, C\right) \\ \mathfrak{r}\left(h_{1}\right) & \text { otherwise }\end{cases}$

$\mathfrak{r}(E h):= \begin{cases}\operatorname{Rep}_{0}, & \text { if } \mathfrak{r}(h) \in \text { Cut } \\ \mathfrak{r}(h), & \text { otherwise }\end{cases}$

$\mathfrak{s}_{n}\left(I_{k, A} h\right):=I_{k, A^{\mathfrak{S}_{n}}}(h)$

$\mathfrak{s}_{n}\left(R_{C} h_{0} h_{1}\right):= \begin{cases}I_{k, \neg c} h_{0}, & \text { if } \mathfrak{r}\left(h_{1}\right)=\left(W_{k}, C\right) \text { and } n=1 \\ R_{C} h_{0} \mathfrak{s}_{n}\left(h_{1}\right), & \text { otherwise }\end{cases}$

$\mathfrak{s}_{n}(E h):= \begin{cases}R_{C} E \mathfrak{s}_{1}(h) E \mathfrak{s}_{0}(h), & \text { if } \mathfrak{r}(h)=(\mathrm{Cut}, C), C \in \mathbb{W}_{0}-F O R \\ R_{C} E \mathfrak{s}_{0}(h) E \mathfrak{s}_{1}(h), & \text { if } \mathfrak{r}(h)=(\mathrm{Cut}, \neg C), C \in \mathbb{W}_{0}-F O R \\ E \mathfrak{s}_{n}(h), & \text { otherwise }\end{cases}$

$\mathfrak{d}\left(I_{k, A} h\right):=\mathfrak{d}(h), \mathfrak{d}\left(R_{C} h_{0} h_{1}\right):=\max \left\{\ell(C), \mathrm{d}\left(h_{0}\right), \mathrm{D}\left(h_{1}\right)\right\}, \mathfrak{D}(E h):=\mathrm{d}(h)-1$,

$\mathfrak{o}\left(I_{k, A} h\right):=\mathfrak{p}(h), \mathfrak{o}\left(R_{C} h_{0} h_{1}\right):=\mathfrak{o}\left(h_{0}\right) \oplus \mathfrak{o}\left(h_{1}\right), \mathfrak{o}(E h):=\exp (\mathfrak{o}(h))$.

3.8 Theorem. For all $h \in Z_{\prec}^{*}$ the following holds:

a) $\mathrm{e}(h) \in S E Q \& \mathrm{r}(h) \in R U L E \& \mathfrak{s}_{n} h \in Z_{\prec}^{*} \&\left(\mathrm{r}(h)=\mathrm{Ax} \Rightarrow \mathfrak{s}_{n} h=h\right)$,

b) $L C\left((\mathrm{r}(h), \mathrm{e}(h)),\left(\mathrm{e}\left(\mathfrak{s}_{n} h\right)\right)_{n \in \mathbb{N}}\right)$, 
c) $\ell_{c}(\mathrm{r}(h)) \leqq \mathrm{d}(h) \& \mathrm{D}\left(\mathfrak{s}_{n} h\right) \leqq \mathrm{D}(h)$,

d) $\mathbf{r}(h) \neq \mathrm{Ax} \Rightarrow \mathrm{p}\left(\mathfrak{s}_{n} h\right) \prec \mathrm{o}(h)$.

Proof by induction on $\ell^{*}(h)$.

The proof of a) is trivial (cf. Lemma 3.5) and one easily verifies that b) and c) hold for all $h \in Z_{<}^{0}$. The induction step for b) and c) is treated in Sect. 5. Here we only carry out the proof of $d$ ).

Proof of 3.8d). Let $Z_{<}^{-}:=\left\{d \in Z_{\prec}: d\right.$ is built up by the rules (Z.1), (Z.4),..,(Z.7) $\}$. Obviously $\mathfrak{o}(d) \prec \exp (1)$, for all $d \in Z_{<}^{-}$, and $\kappa[A] \in Z_{<}^{-}$, for all $A \in \mathrm{FOR}$.

(0.1) $h=\langle($ Ind, $F), \Gamma, \emptyset\rangle$ : Then $\mathfrak{s}_{n} h=c_{n}^{F} \in Z_{\prec}^{-}$and thus $\mathrm{o}\left(c_{n}^{F}\right)\langle\exp (1)=\mathrm{o}(h)$.

(0.2) $h=\langle(\mathrm{TI}, \boldsymbol{F}, \boldsymbol{m}), \Gamma, \emptyset\rangle$ :

$(0.2 .1) n \prec m$ : Then $s_{n} h=\left\langle\left(W_{1}, \cdot\right), \cdot,\left\langle\left(W_{n}, \cdot\right), \cdot\langle(\mathbb{M}, \cdot), \cdot,(e, \kappa[F(n)])\rangle\right\rangle\right\rangle$ with $e=\langle(\mathrm{TI}, F, n), \cdot, \emptyset\rangle, \mathfrak{o}(\kappa[F(n)])<\exp (m), \mathrm{o}(e)=\prec-\max \{\exp (n), 1 \oplus 1\}<\exp (m)$.

Hence $\mathfrak{o}\left(\mathfrak{s}_{n} h\right)=\langle-\max \{\mathfrak{o}(e) \oplus 1, \mathfrak{o}(\kappa[F(n)]) \oplus 1\} \oplus 1 \oplus 1<\exp (m)=\mathfrak{v}(h)$.

(0.2.1) $n \nless m$ : Then $\mathfrak{s}_{n} h=\left\langle\left(\mathrm{W}_{0}, \cdot\right), \cdot,\langle\mathrm{Ax}, \cdot, \boldsymbol{\emptyset}\rangle\right\rangle$ and therefore $\mathrm{o}\left(\mathfrak{s}_{n} h\right)=1\langle\oplus \preceq \mathrm{o}(h)$.

(0.3) $h=\left\langle(v, \forall x F(x)), \cdot, d_{0}\right\rangle$ : Then $\mathfrak{s}_{n} h=d_{0}[v / n]$ and $\mathfrak{o}\left(d_{0}[v / n]\right)=\mathfrak{o}\left(d_{0}\right) \prec \mathfrak{v}\left(d_{0}\right) \oplus 1$ $=\mathfrak{o}(h)$.

(0.4) otherwise: trivial.

(1) $h=I_{k, A} g$ : We have $\mathfrak{o}(h)=\mathfrak{o}(g), \mathfrak{o}\left(\mathfrak{s}_{n} h\right)=\mathfrak{v}\left(I_{k, A^{\mathfrak{s}} n} g\right)=\mathfrak{v}\left(\mathfrak{s}_{n} g\right)$, and by I.H. $\mathfrak{v}\left(\mathfrak{s}_{n} g\right)$ $\prec \mathbf{v}(\mathrm{g})$.

(2) $h=R_{C} h_{0} h_{1}$ : We have $\mathfrak{o}(h)=\mathfrak{o}\left(h_{0}\right) \oplus \mathfrak{o}\left(h_{1}\right),\left(\mathfrak{s}_{n} h=I_{k} \neg c h_{0}\right.$ or $\left.\mathfrak{s}_{n} h=R_{C} h_{0} \mathfrak{s}_{n} h_{1}\right)$, $\mathfrak{o}\left(I_{k, \neg C} h_{0}\right)=\mathfrak{o}\left(h_{0}\right) \leqq \mathfrak{o}\left(h_{0}\right) \oplus \mathfrak{o}\left(\mathfrak{s}_{n} h_{1}\right)=\mathfrak{o}\left(R_{C} h_{0} \mathfrak{s}_{n} h_{1}\right)$ and (by I.H.) $\mathfrak{o}\left(\mathfrak{s}_{n} h_{1}\right)<\mathfrak{v}\left(h_{1}\right)$.

(3) $h=E g$ : It suffices to consider the case where $\mathfrak{s}_{h} h=R_{A}\left(E_{\mathfrak{s}_{1}} g\right)\left(E_{\mathfrak{s}_{0}} g\right)$.

Then we have $\mathfrak{d}\left(\mathfrak{s}_{h} h\right)=\exp \left(\mathfrak{d}\left(\mathfrak{s}_{1} g\right)\right) \oplus \exp \left(\mathfrak{d}\left(\mathfrak{s}_{0} g\right)\right)$ and (by I.H.) $\mathfrak{d}\left(\mathfrak{s}_{0} g\right), \mathfrak{d}\left(\mathfrak{s}_{1} g\right) \prec \mathfrak{p}(g)$. This yields $\mathfrak{p}\left(\mathfrak{s}_{n} h\right)<\exp (\mathfrak{o}(g))=\mathfrak{v}(h)$.

\section{Proof theoretic analysis of $Z+T I_{<\mid}$}

Now we carry out the proof theoretic analysis of $Z+T I_{<}$sketched at the beginning of Sect. 3. Let $p \in L$ be a fixed binary predicate symbol and $\mathbf{p} \subseteq \mathbb{N}^{2}$ the primitive recursive relation assigned to $p$.

Abbreviation: $A: \equiv \forall x \exists y p x y, \mathrm{f}_{p}(n):=\min \{m: \mathbf{p}(n, m)\}$.

4.1 Definition of prim. rec. functions sub: $Z_{\prec}^{*} \rightarrow Z_{\prec}^{*}, \wedge: Z_{\prec}^{*} \rightarrow Z_{\prec}^{*}$

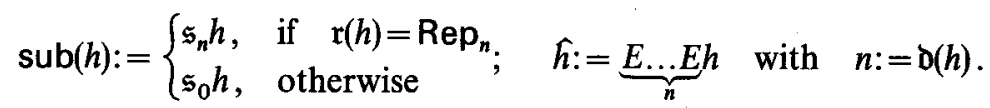

4.2 Lemma. For every $h \in Z_{\prec}^{*}$ holds:

a) $\mathrm{D}(\hat{h})=0$.

b) $\mathfrak{r}(h) \neq \mathrm{Ax} \Rightarrow \mathrm{v}(\operatorname{sub}(h))<\mathfrak{v}(h), \mathfrak{r}(h)=\mathrm{Ax} \Rightarrow \operatorname{sub}(h)=h$.

Proof. Trivial. (cf. definition of $D$ and Theorem 3.7a), d)).

4.3 Definition of prim. rec. functions $s: Z_{\prec}^{0} \times \mathbb{N}^{2} \rightarrow Z_{\prec}^{*}, \mathrm{u}: S E Q \rightarrow \mathbb{N}$ $\mathrm{s}(d, n, k):=\operatorname{sub}^{(k)}\left(I_{n, A} d\right)$ (where sub ${ }^{(k)}$ is the $k$ th iterate of sub). $\mathrm{u}(\Gamma):=\sup \{m: m$ occurs in $\Gamma\}$. 


\subsection{Lemma}

$d \in Z_{\prec}^{0} \& \mathrm{e}(d) \subseteq\{A\} \& \mathrm{o}(\mathrm{s}(d, n, k+1)) \nless \mathrm{o}(\mathrm{s}(d, n, k))$

$\Rightarrow \exists I I I \mathbf{u}(\mathbf{e}(\mathbf{s}(d, n, k))) \mathbf{p}(n, m)$

Proof. Abb.: $h_{n, k}:=\mathrm{s}(d, n, k), \Gamma(n):=\{\exists y p n y\} \cup\{p n m: m \in \mathbb{N}\}$.

Then we obtain

(1) $h_{n, 0} \in Z_{\prec}^{*} \wedge \mathrm{D}\left(h_{n, 0}\right)=0 \wedge \mathfrak{e}\left(h_{n, 0}\right) \subseteq \Gamma(n)$ [by definition of $\mathfrak{d}$ and $\mathfrak{e}$ ]

(2) $h_{n, k} \in Z_{\prec}^{*} \wedge \mathfrak{D}\left(h_{n, k}\right)=0[(1)$, Theorem 3.7a), c), induction on $k]$

(3) $\mathfrak{e}\left(h_{n, k}\right) \subseteq \Gamma(n) \Rightarrow \mathrm{r}\left(h_{n, k}\right) \in\left\{A x, \operatorname{Rep}_{i},\left(W_{i}, \exists y p n y\right)\right\}[(2)$, Theorem 3.7b)]

(4) $\mathfrak{e}\left(h_{n, k}\right) \subseteq \Gamma(n) \Rightarrow \mathrm{e}\left(h_{n, k+1}\right) \subseteq \Gamma(n)[(3)$, Theorem 3.7b)]

(5) $\mathfrak{e}\left(h_{n, k}\right) \subseteq \Gamma(n)[(4)$, induction on $k]$

(6) $\left.\mathfrak{r}\left(h_{n, k}\right)=\mathrm{Ax} \Rightarrow \operatorname{II} m\left(p n m \in \mathfrak{e}\left(h_{n, k}\right) \& p n m \in T R U E\right)\right)$ [(5), Theorem 3.7b)]

(7) $\mathfrak{r}\left(h_{n, k}\right)=\mathrm{Ax} \Rightarrow \operatorname{II} m \leqq \mathrm{u}\left(\mathrm{e}\left(h_{n, k}\right)\right) \mathrm{p}(n, m)[(6)]$

From (7) the assertion follows by Lemma $4.2 \mathrm{~b}$ ).

4.5 Theorem. If $Z+T I_{\prec \uparrow} \vdash \forall x \exists y p x y$ then there are prim. rec. functions $\mathrm{g}$, $0: \mathbb{N}^{2} \rightarrow \mathbb{N}, \mathrm{q}: \mathbb{N} \rightarrow Z_{<}^{*}, \mathrm{~g}^{\prime}: Z_{\prec}^{*} \rightarrow \mathbb{N}$ and an $\mathrm{a} \in \mathscr{A}$, such that, for all $n \in \mathbb{N}:$

a) $\mathrm{f}_{p}(n)=\mathrm{g}(n, \min \{k: \mathrm{o}(n, k+1) \nless \mathrm{o}(n, k)\})$ and $\forall k(\mathrm{o}(n, k) \leqq a)$.

b) $\mathrm{f}_{p}(n)=\mathscr{F}(\mathrm{q}(n))$, where

$$
\mathscr{F}(h):= \begin{cases}\mathscr{F}(\operatorname{sub}(h)), & \text { if } \mathfrak{o}(\operatorname{sub}(h))<\mathfrak{v}(h) \leqq a \\ g^{\prime}(h), & \text { otherwise }\end{cases}
$$

Proof. By assumption there exists a $d \in Z_{\prec}^{0}$ with $\mathrm{e}(d) \subseteq\{A\}$.

a) We set $\mathrm{o}(n, k):=\mathrm{o}(\mathrm{s}(d, n, k)), \mathrm{g}(n, k):=\min \{m \leqq \mathrm{u}(\mathrm{e}(\mathrm{s}(d, n, k))): \quad \mathbf{p}(n, m)\}$, $\mathrm{a}:=\mathfrak{o}(\hat{d})$. By definition of $\mathfrak{v}$ and Lemma $4.2 \mathrm{~b})$ we get $\forall n, k(\mathrm{o}(n, k) \leqq \mathrm{o}(n, 0)=\mathrm{a})$. Since $\prec$ is wellfounded, we have $\forall n$ II $k(o(n, k+1) \nless o(n, k))$. By Lemma 4.4 this yields the assertion.

b) Let $\mathrm{q}(n):=\mathrm{s}(d, n, 0)=I_{n, A} \hat{d}$ and $\mathrm{g}^{\prime}(h):=\min \{m \leqq \mathrm{u}(\mathrm{e}(h)): \mathrm{p}(\mathrm{n}(h), m)\}$, where

$$
\mathrm{r}(h):=\left\{\begin{array}{ll}
n, & \text { if } h=I_{n, A} h_{0} \\
0, & \text { if } h \text { has not the form } I_{n, A} h_{0}
\end{array} .\right.
$$

Obviously $\mathscr{F}(\mathrm{q}(n))=\mathrm{g}^{\prime}\left(h_{n}^{*}\right)$ with $h_{n}^{*}=\mathrm{s}\left(d^{\prime}, n, k_{n}\right), k_{n}:=\min \{k: \mathrm{o}(n, k+1) \nless \mathrm{o}(n, k)\}$. Since $\forall i \in \mathbb{N}, h \in Z_{\prec}^{*}\left(\mathfrak{s}_{i} I_{n, A} h=I_{n, A} \mathfrak{s}_{i} h\right)$, we have $\mathrm{n}\left(h_{n}^{*}\right)=n$ and thus

$$
\mathrm{g}^{\prime}\left(h_{n}^{*}\right)=\min \left\{m \leqq \mathrm{u}\left(\mathrm{e}\left(h_{n}^{*}\right)\right): \mathbf{p}(n, m)\right\}=\mathrm{g}\left(n, k_{n}\right) .
$$

But $g\left(n, k_{n}\right)=\mathrm{f}_{p}(n)$ by a).

Now we assume that the elementary properties of $(\mathscr{A}, \prec, \oplus$, exp), i.e. $(\prec .1)-(\prec .4)$ on p. 280 and the transitivity of $\prec$, are provable in $P R A$. Then the proof of Lemma 4.4 can be formalized in $P R A$, and we obtain the following theorem.

4.6 Theorem $\left(P R A \vdash P R W O(\prec) \rightarrow \Pi_{2}^{0}\right.$-Reflection $\left.\left(Z+T I_{<1}\right)\right)$. There exists a primitive recursive function $\tilde{\mathrm{o}}: \mathbb{N}^{2} \rightarrow \mathbb{N}$ such that

$$
P R A \vdash W F(\tilde{0}, \prec) \rightarrow\left(\operatorname{Prov}_{Z+T I_{<\uparrow}}(\ulcorner C\urcorner) \rightarrow C\right),
$$

for each $\Pi_{2}^{0}$-sentence $C$ of $L$, where

$$
W F(\tilde{\mathrm{o}}, \prec): \equiv \forall x \exists y(\tilde{\mathrm{o}}(x, y+1) \nless \tilde{\mathrm{o}}(x, y)),
$$

and $\operatorname{Prov}_{Z+T I<\uparrow}(\ulcorner C\urcorner)$ formalizes the statement " $\exists l d \in Z_{\prec}^{0}(\operatorname{End}(d) \leqq\{C\})$ ". 
Proof. Let

$$
\tilde{\mathrm{o}}(j, k):=\left\{\begin{array}{ll}
\mathrm{o}\left(\mathrm{sub}^{(k)}(h)\right), & \text { if } j \text { is the Gödelnumber of } h \in Z_{\prec}^{*} \\
0, & \text { otherwise }
\end{array} .\right.
$$

Without loss of generality we may assume that $C \equiv \forall x \exists y p x y$.

Then by Lemma 4.4 we have

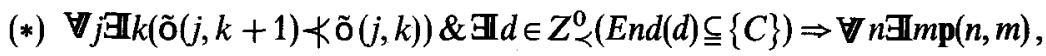

and therefore the assertion is obtained by formalizing in $P R A$ the proof of 4.4 .

Corollary. $Z+T I_{<\uparrow} \nvdash W F(\tilde{o}, \prec)$.

Proof. By 4.6

i.e.

$$
\left.Z+T I_{\prec \uparrow} \vdash W F(\tilde{\mathrm{o}}, \prec) \rightarrow\left(\operatorname{Prov}_{Z+T I_{<\uparrow}}(\mathrm{r} 0=1\urcorner\right) \rightarrow 0=1\right),
$$

$$
Z+T I_{\prec \uparrow} \vdash W F(\tilde{0}, \prec) \rightarrow \operatorname{CON}\left(Z+T I_{\prec \uparrow}\right) .
$$

Hence $Z+T I_{\prec \uparrow} \nvdash W F(\tilde{0}, \prec)$ by Gödel's Second Incompleteness Theorem.

\section{Arbitrary notation systems for derivations}

In this section we analyze the construction of the $\operatorname{system}\left(Z_{\prec}^{*}, \mathfrak{e}, \mathfrak{r}, \mathfrak{s}\right)$ in a somewhat more general context. We introduce the notion of an arbitrary notation system (for elements of TREE) and define the *-extension $\mathscr{H}^{*}$ of an arbitrary notation system $\mathscr{H}$ literally in the same way as we have defined $\left(Z_{<}^{*}, \mathfrak{e}, \mathfrak{x}, \mathfrak{s}\right)$ from $\left(Z_{\prec}^{0}, \mathfrak{e}, \mathfrak{r}, \mathfrak{s}\right)$ in Sect. 3. This will then be used in Sect. 6 to give an alternative description of Minc's continuous cut-elimination operator $\mathscr{E}^{\prime}$ for arbitrary (not necessarily wellfounded) proof-figures.

5.1 Definition. A notation system consists of a nonempty set $H$ and functions $\mathfrak{q}: H \rightarrow R U L E \times S E Q, \mathfrak{s}: \mathbb{N} \times H \rightarrow H$ such that $\forall(n, h) \in \mathbb{N} \times H\left(\mathfrak{q}(h) \in \mathrm{Ax} \Rightarrow \mathfrak{s}_{n} h=h\right)$.

5.2 Definitions. Let $\mathscr{H}=(H, \mathfrak{q}, \mathfrak{s})$ be a notation system and $h \in H$ :

a) $\mathfrak{r}(h):=\mathfrak{q}^{0}(h):=\mathbf{r}, \mathfrak{e}(h):=\mathfrak{q}^{1}(h):=\Gamma$, where $(\mathbf{r}, \Gamma):=\mathfrak{q}(h)$.

b) $\left[h,\left\langle n_{0}, \ldots, n_{k-1}\right\rangle\right]:=\mathfrak{s}_{n_{k-1}} \ldots \mathfrak{s}_{n_{0}} h$

c) $\{h\}: \mathbb{N}^{<\omega} \rightarrow R U L E \times S E Q,\{h\}(\sigma):=\mathfrak{q}([h, \sigma])$

d) $\mathscr{H}$ is correct $: \Leftrightarrow(\mathbb{V} h \in H) L C_{\mathscr{H}}(h)$, with $L C_{\mathscr{H}}(h): \Leftrightarrow L C\left(\mathfrak{q}(h),\left(\mathfrak{e}\left(\mathfrak{s}_{n} h\right)\right)_{n \in \mathbb{N}}\right)$.

e) $\mathrm{D}: H \rightarrow \mathbb{N}$ is a cut-bound for $\mathscr{H}: \Leftrightarrow \forall h \in H \forall n\left[\ell_{c}(\mathfrak{r}(h)) \leqq \mathfrak{d}(h) \& \mathrm{~d}\left(\mathfrak{s}_{n} h\right) \leqq \mathrm{d}(h)\right]$.

5.3 Theorem. Let $\mathscr{H}=(H, \mathfrak{q}, \mathfrak{s})$ be a notation system.

a) For all $h \in H, n \in \mathbb{N}, \sigma \in \mathbb{N}^{<\omega}$ we have $\{h\} \in T R E E, E n d(\{h\})=\mathrm{e}(h)$, Rule $(\{h\})=\mathfrak{r}(h),\left\{\mathfrak{s}_{n} h\right\}=\{h\}[n],\{[h, \sigma]\}=\{h\} \llbracket \sigma \rrbracket$.

b) $\mathscr{H}$ is correct $\Leftrightarrow \forall h \in H(\{h\}$ is locally correct $)$

c) If $\mathrm{D}$ is a cut-bound for $\mathscr{H}$, then $\forall h \in H(\operatorname{deg}(\{h\}) \leqq \mathrm{D}(h))$.

d) $h \in H \& \mathrm{q}(h) \in \mathrm{Ax} \Rightarrow\{h\}=\mathbf{0}_{\mathrm{q}(h)}$.

Proof. a)

(1) $\{h\}(\sigma) \in \mathrm{Ax} \Rightarrow \mathrm{q}([h, \sigma]) \in \mathrm{Ax} \Rightarrow \forall n\left(\mathfrak{s}_{n}[h, \sigma]=[h, \sigma]\right)$

$$
\begin{aligned}
& \Rightarrow \mathbb{V} n\left(\{h\}(\sigma *\langle n\rangle)=\mathfrak{q}([h, \sigma *\langle n\rangle])=\mathfrak{q}\left(\mathfrak{s}_{n}(h, \sigma]\right)\right. \\
& =\mathfrak{q}([h, \sigma])=\{h\}(\sigma)) . \quad \text { Therefore }\{h\} \in T R E E .
\end{aligned}
$$


(2) $($ Rule $(\{h\}), \operatorname{End}(\{h\}))=\{h\}(\langle\rangle)=\mathfrak{q}(h)=(\mathfrak{r}(h), \mathfrak{e}(h))$.

(3) $\left\{\mathfrak{s}_{n} h\right\}(\sigma)=\mathfrak{q}\left(\left[\mathfrak{s}_{n} h, \sigma\right]\right)=\mathfrak{q}([h,\langle n\rangle * \sigma])=\{h\}(\langle n\rangle * \sigma)=(\{h\}[n])(\sigma)$.

(4) $\{[h, \sigma]\}=\{h\} \llbracket \sigma \rrbracket$ follows from (3) by induction on the length of $\sigma$.

b) Using $\mathfrak{e}\left(\mathfrak{s}_{n}[h, \sigma]\right)=\{h\}^{1}(\sigma *\langle n\rangle)$ we obtain

$$
\begin{aligned}
& \forall h . L C_{\mathscr{H}}(h) \Leftrightarrow \forall h . L C\left(\mathfrak{q}(h),\left(\mathrm{e}\left(\mathfrak{s}_{n} h\right)\right)_{n \in \mathbf{N}}\right) \\
&\left.\Leftrightarrow \forall h \forall \sigma . L C\left(\mathfrak{q}([h, \sigma]),\left(\mathfrak{e}\left(\mathfrak{s}_{n} H h, \sigma\right]\right)\right)_{n \in \mathbb{N}}\right) \\
& \Leftrightarrow \forall h \forall \sigma . L C\left(\{h\}(\sigma),\left(\{h\}^{1}(\sigma *\langle n\rangle)\right)_{n \in \mathbb{N}}\right) \\
& \Leftrightarrow \forall h(\{h\} \text { locally correct). } \\
& \text { c) }\{h\}^{0}(\sigma)=(\text { Cut, } A) \Rightarrow \mathfrak{r}([h, \sigma])=(\text { Cut }, A) \Rightarrow \ell(A)<\mathrm{d}([h, \sigma]) \leqq \mathcal{D}(h) . \\
& \text { d) }\{h\}(\sigma)=\mathfrak{q}([h, \sigma])=\mathfrak{q}(h) . \quad \square
\end{aligned}
$$

5.4 Definition and Remark. Let $\mathscr{H}=(H, \mathfrak{q}, \mathfrak{s})$ be a notation system.

a) The mapping $\{\cdot\}: H \rightarrow T R E E, h \mapsto\{h\}$ is called the canonical interpretation for $\mathscr{H}$. If

$$
\mathbf{j}: H \rightarrow T R E E \quad \text { with } \quad \forall h \in H \forall n\left(\mathbf{j}(h)\left(\langle>)=\mathfrak{q}(h) \& \mathrm{j}\left(\mathfrak{s}_{n} h\right)=\mathrm{j}(h)[n]\right)\right.
$$

then

$$
\mathrm{j}(h)(\sigma)=(\mathrm{j}(h) \llbracket \sigma])(\langle\rangle)=\mathrm{j}([h, \sigma])(\langle\rangle)=\mathrm{q}([h, \sigma])=\{h\}(\sigma)
$$

and therefore $\mathbf{j}=\{\cdot\}$.

b) $\mathscr{H}$ is called wellfounded iff $\forall h \in H(\{h\} \in W T)$. If $\mathscr{H}$ is wellfounded then $\|\{h\}\| \in O n$ is defined for all $h \in H$, and by 5.3a), we have $\mathfrak{r}(h) \neq \mathrm{Ax} \Rightarrow \forall n\left(\left\|\left\{\mathfrak{s}_{n} h\right\}\right\|\right.$ $<\|\{h\}\|)$.

5.5 Definition of the notation system $\mathscr{H}^{*}$. Let $\mathscr{H}=(H, \mathfrak{q}, \mathfrak{s})$ be a notation system.

$H^{*}:=\left\{\begin{array}{l}\text { set of all terms } h \text { generated from constants } d \in H \text { by the } \\ \text { function symbols } I_{k, A}, R_{C}, E\left(k \in \mathbb{N}, A \in \mathbb{A}-F O R, C \in W_{0^{-}} F O R\right) .\end{array}\right.$

For $h \in H^{*}$ we define $\ell^{*}(h)$ as in 3.3 .

Then the functions $\mathfrak{e}=\mathfrak{q}^{1}: H \rightarrow S E Q, \mathfrak{x}=\mathfrak{q}^{0}: H \rightarrow R U L E, \mathfrak{s}: \mathbb{N} \times H \rightarrow H$ are extended to functions $\mathrm{e}: H^{*} \rightarrow S E Q, \mathfrak{x}: H^{*} \rightarrow R U L E, \mathfrak{s}: \mathbb{N} \times H^{*} \rightarrow H^{*}$ precisely as in Sect. 3, i.e. by Def. 3.7.

$\mathscr{H}^{*}:=\left(H^{*}, \mathfrak{q}, \mathfrak{s}\right)$ with the extended functions $\mathfrak{q}=(\mathrm{r}, \mathfrak{e})$ and $\mathfrak{s}$.

We call $\mathscr{H}^{*}$ the *-extension of $\mathscr{H}$.

5.6. Remark. The canonical interpretation $\{\cdot\}^{*}: \mathscr{H}^{*} \rightarrow T R E E,\{h\}^{*}:=\lambda \sigma . \mathrm{q}([h, \sigma])$ is of course an extension of the canonical interpretation $\{\cdot\}: \mathscr{H} \rightarrow T R E E$. So we may drop the superscript $*$ as we have already done for $\mathfrak{e}, \mathfrak{r}, \mathfrak{s}$.

\subsection{Theorem}

a) $\mathscr{H}$ correct $\Rightarrow \mathscr{H}^{*}$ correct

b) Every cut-bound $\mathrm{D}$ for $\mathscr{H}$ can be extended to a cut-bound for $\mathscr{H}^{*}$ by

$$
\grave{D}\left(I_{k, A} h\right):=\mathfrak{D}(h), \mathfrak{d}\left(R_{C} h_{0} h_{1}\right):=\max \left\{\ell(C), \mathfrak{D}\left(h_{0}\right), \mathfrak{D}\left(h_{1}\right)\right\}, \mathfrak{D}(E h):=\mathrm{d}(h)-1 .
$$

c) $\mathscr{H}$ wellfounded $\Rightarrow \mathscr{H}^{*}$ wellfounded.

Proof. a) and b) are proved by (quantifierfree) induction on $\ell^{*}(h)$. The proof is just a straightforward verification. Nevertheless we carry it out in full detail, since it is 
one of the main proofs of this paper, and in addition we want to demonstrate that even such a detailed exposition is possible without excessive effect.

a) Let $\mathfrak{U}(h, n)$ abbreviate the conjunction of $(1)-(5)$.

(1) $\mathfrak{r}(h)=\mathrm{Ax} \Rightarrow \mathrm{e}(h) \cap T R U E \neq \emptyset$,

(2) $\mathfrak{r}(h)=(M, A) \Rightarrow A \in \mathfrak{e}(h) \& \mathfrak{e}\left(\mathfrak{s}_{n} h\right) \subseteq \mathfrak{e}(h) \cup A[n]$,

(3) $\mathfrak{r}(h)=\left(W_{k}, A\right) \Rightarrow A \in \mathfrak{e}(h) \& \mathfrak{e}\left(\mathfrak{s}_{0} h\right) \subseteq \mathfrak{e}(h) \cup A[k]$,

(4) $\mathrm{r}(h)=($ Cut, $C) \Rightarrow \mathrm{e}\left(\mathfrak{s}_{0} h\right) \cong \mathrm{e}(h) \cup C \& \mathrm{e}\left(\mathfrak{s}_{1} h\right) \cong \mathrm{e}(h) \cup \neg C$,

(5) $\mathfrak{r}(h)=\operatorname{Rep}_{n} \Rightarrow \mathrm{e}\left(\mathfrak{s}_{n} h\right) \subseteq \mathfrak{e}(h)$.

Obviously

and

$$
\forall h \in H\left[L C_{\mathscr{H}}(h) \Leftrightarrow \forall n \mathfrak{R}(h, n)\right]
$$

$$
\forall h \in H^{*}\left[L C_{\mathscr{H}^{*}}(h) \Leftrightarrow \forall n \mathfrak{P}(h, n)\right] .
$$

So we have to prove $\forall h \in H^{*} \mathfrak{A}(h, n)$ under the premise $\forall h \in H \mathfrak{A}(h, n)$.

This will be done by induction on $\ell^{*}(h)$.

If $\ell^{*}(h)=0$ then $h \in H$, and we are done. - Now let $\ell^{*}(h)>0$.

I. $h=I_{m, B} h_{1}$ - Then $\mathrm{e}(h)=\left(\mathrm{e}\left(h_{1}\right) \backslash B\right) \cup B[m]$ and $\mathfrak{s}_{n} h=I_{m, B} \mathfrak{s}_{n} h_{1}$.

I.1 $\mathfrak{r}\left(h_{1}\right)=(\mathrm{A}, B)$ : Then $\mathfrak{r}(h)=\operatorname{Rep}_{m}$ and (1) (4) are trivially true. To prove (5) we assume that $\mathfrak{r}(h)=\operatorname{Rep}_{n}$. Then $n=m$ and (by I.H.) $\mathfrak{e}\left(\mathfrak{s}_{n} h_{1}\right) \subseteq \mathfrak{e}\left(h_{1}\right) \cup B[n]$. This yields

$$
\mathfrak{e}\left(\mathfrak{s}_{n} h\right)=\left(\mathfrak{e}\left(\mathfrak{s}_{n} h_{1}\right) \backslash B\right) \cup B[m] \subseteq\left(\mathfrak{e}\left(h_{1}\right) \backslash B\right) \cup B[m]=\mathfrak{e}(h) .
$$

I.2 $\mathfrak{r}\left(h_{1}\right)=(M, A)$ with $A \not \equiv B$ : Then $\mathfrak{r}(h)=\mathfrak{r}\left(h_{1}\right)$ and (by I.H.)

Hence

$$
A \in \mathrm{e}\left(h_{1}\right) \& \mathrm{e}\left(\mathfrak{s}_{n} h_{1}\right) \subseteq \mathrm{e}\left(h_{1}\right) \cup A[n] .
$$

$$
\begin{aligned}
\mathrm{e}\left(\mathfrak{s}_{n} h\right) & =\left(\mathfrak{e}\left(\mathfrak{s}_{n} h_{1}\right) \backslash B\right) \cup B[m] \leqq\left(\mathrm{e}\left(h_{1}\right) \backslash B\right) \cup B[m] \cup A[n] \\
& =\mathrm{e}(h) \cup A[n],
\end{aligned}
$$

and $A \in \mathrm{e}\left(h_{1}\right) \backslash B \subseteq \mathrm{e}(h)$.

I.3 $\mathfrak{r}\left(h_{1}\right)=\left(\mathrm{W}_{k}, A\right)$, (Cut, $\left.A\right), \operatorname{Rep}_{k}:$ as I.2.

II. $h=R_{C} h_{0} h_{1}$. By definition $\mathrm{e}(h)=\Gamma_{0} \cup\left(\mathfrak{e}\left(h_{1}\right) \backslash C\right)$ with $\Gamma_{0}:=\mathfrak{e}\left(h_{0}\right) \backslash \neg C$.

II.1 $\mathfrak{r}\left(h_{1}\right)=\left(W_{k}, C\right)$ : Then $\mathfrak{r}(h)=($ Cut, $C[k]), \mathfrak{s}_{0} h=R_{C} h_{0} \mathfrak{s}_{0} h_{1}, \mathfrak{s}_{1} h=I_{k}, \neg c h_{0}$, and (by I.H.) $\mathrm{e}\left(\mathfrak{s}_{0} h_{1}\right) \subseteq \mathrm{e}\left(h_{1}\right) \cup C[k]$. Hence

$$
\begin{aligned}
\mathfrak{e}\left(\mathfrak{s}_{0} h\right) & =\mathfrak{e}\left(R_{C} h_{0} \mathfrak{s}_{0} h_{1}\right)=\Gamma_{0} \cup\left(\mathfrak{e}\left(\mathfrak{s}_{0} h_{1}\right) \backslash C\right) \subseteq \Gamma_{0} \cup\left(\mathfrak{e}\left(h_{1}\right) \backslash C\right) \cup C[k] \\
& =\mathfrak{e}(h) \cup C[k]
\end{aligned}
$$

and

$$
\mathfrak{e}\left(\mathfrak{s}_{1} h\right)=\mathrm{e}\left(I_{k, \neg C} h_{0}\right)=\left(\mathfrak{e}\left(h_{0}\right) \backslash \neg C\right) \cup \neg C[k] \subseteq \mathfrak{e}(h) \cup \neg C[k] .
$$

II.2 $\mathfrak{r}\left(h_{1}\right)=(\mathbb{M}, A)$ : Then $\mathfrak{r}(h)=(\mathbb{X}, A), A \not \equiv C, \mathfrak{s}_{n} h=R_{C} h_{0} \mathfrak{s}_{n} h_{1}$ and (by I.H.)

$$
A \in \mathfrak{e}\left(h_{1}\right) \& \mathrm{e}\left(\mathfrak{s}_{n} h_{1}\right) \subseteq \mathfrak{e}\left(h_{1}\right) \cup A[n]
$$

Hence $A \in \mathrm{e}(h)$ and

$$
\mathfrak{e}\left(\mathfrak{s}_{n} h\right)=\Gamma_{0} \cup\left(\mathfrak{e}\left(\mathfrak{s}_{n} h_{1}\right) \backslash C\right) \subseteq \Gamma_{0} \cup\left(\mathfrak{e}\left(h_{1}\right) \backslash C\right) \cup A[n]=\mathfrak{e}(h) \cup A[n] .
$$

II.3 $\mathfrak{r}\left(h_{1}\right)=($ Cut, $A)$ or $\operatorname{Rep}_{k}$ : Then $\mathfrak{r}(h)=\mathfrak{r}\left(h_{1}\right)$, and $\mathfrak{U}(h, n)$ follows immediately from the I.H.

II.4 $\mathfrak{r}\left(h_{1}\right)=\mathrm{Ax}$ : Then also $\mathfrak{r}(h)=\mathrm{Ax}$, and by I.H. we have $\mathrm{e}\left(h_{1}\right) \cap T R U E \neq \emptyset$. From this we obtain $\mathrm{e}(h) \cap T R U E \neq \emptyset$, since $C \notin T R U E$. 
III.1 $h=E h_{1}$ and $\mathfrak{r}\left(h_{1}\right)=(C u t, C)$ : w.l.o.g. $C \in \mathbb{W}_{0^{-F O R}}$. Then $\mathfrak{r}(h)=\operatorname{Rep}_{0}$, $\mathfrak{s}_{0} h=R_{C}\left(E \mathfrak{s}_{1} h_{1}\right)\left(E \mathfrak{s}_{0} h_{1}\right)$ and

$$
\mathfrak{e}\left(\mathfrak{s}_{0} h_{1}\right) \subseteq \mathfrak{e}\left(h_{1}\right) \backslash C \& \mathrm{e}\left(\mathfrak{s}_{1} h_{1}\right) \subseteq \mathfrak{e}\left(h_{1}\right) \backslash \neg C .
$$

Hence

$$
\mathrm{e}\left(\mathfrak{s}_{0} h\right)=\left(\mathrm{e}\left(\mathfrak{s}_{1} h_{1}\right) \backslash \neg C\right) \cup\left(\mathrm{e}\left(\mathfrak{s}_{0} h_{1}\right) \backslash C\right) \cong \mathrm{e}\left(h_{1}\right)=\mathrm{e}(h) .
$$

III.2 $h=E h_{1}$ and $\mathfrak{r}\left(h_{1}\right) \notin$ Cut: Then $\mathfrak{r}(h)=\mathfrak{r}\left(h_{1}\right)$ and $\mathfrak{s}_{n} h=E \mathfrak{s}_{n} h_{1}$. This together with $\mathfrak{e}(h)=\mathfrak{e}\left(h_{1}\right), \mathfrak{e}\left(\mathfrak{s}_{n} h\right)=\mathfrak{e}\left(\mathfrak{s}_{n} h_{1}\right)$ and the I.H. yields the assertion $\mathfrak{A}(h, n)$.

b) We prove

$$
\mathfrak{d}\left(\mathfrak{s}_{n} h\right) \leqq \mathfrak{d}(h) \&(\mathfrak{r}(h)=(\text { Cut }, A) \Rightarrow \ell(A)<\mathfrak{D}(h))
$$

by induction on $\ell^{*}(h)$.

I. $h=I_{m, b} h_{1}$. By I.H. we obtain $\mathrm{d}\left(\mathfrak{s}_{n} h\right)=\mathrm{d}\left(\mathfrak{s}_{n} h_{1}\right) \leqq \mathrm{d}\left(h_{1}\right)=\mathrm{d}(h)$ and

$$
\mathfrak{r}(h)=(\text { Cut, } A) \Rightarrow \mathfrak{r}\left(h_{1}\right)=(\text { Cut }, A) \Rightarrow \ell(A)<\mathfrak{D}\left(h_{1}\right)=\mathfrak{d}(h) \text {. }
$$

II. $h=R_{C} h_{0} h_{1}$. Then $\mathfrak{d}(h)=\max \left\{\ell(C), \mathfrak{D}\left(h_{0}\right), \mathfrak{D}\left(h_{1}\right)\right\}$ and (by I.H.) $\mathfrak{d}\left(\mathfrak{s}_{\mathrm{n}} h_{1}\right) \leqq \mathfrak{d}\left(h_{1}\right)$.

II.1 $\mathfrak{r}\left(h_{1}\right)=\left(W_{k}, C\right)$ : Then $\mathfrak{r}(h)=($ Cut, $C[k])$ and $\ell(C[k])<\ell(C) \leqq \mathfrak{d}(h)$.

II.1.1 $n=1$ : Then $\mathfrak{s}_{n} h=I_{k, \neg c} h_{0}$ and therefore $\mathfrak{d}\left(\mathfrak{s}_{n} h\right)=\mathfrak{d}\left(h_{0}\right) \leqq \mathfrak{d}(h)$

II.1.2 $n \neq 1$ : Then $\mathfrak{s}_{n} h=R_{C} h_{0} \mathfrak{s}_{n} h_{1}$ and therefore

$$
\mathfrak{D}\left(\mathfrak{s}_{n} h\right)=\max \left\{\ell(C), \mathfrak{D}\left(h_{0}\right), \mathfrak{D}\left(\mathfrak{s}_{n} h_{1}\right)\right\} \leqq \max \left\{\ell(C), \mathfrak{D}\left(h_{0}\right), \mathfrak{D}\left(h_{1}\right)\right\}=\mathfrak{D}(h) .
$$

II.2 $\mathfrak{r}\left(h_{1}\right) \neq\left(\mathrm{W}_{k}, C\right)$ : Then also $\mathfrak{s}_{n} h=R_{C} h_{0} \mathfrak{s}_{n} h_{1}$ and thus $\mathrm{D}\left(\mathfrak{s}_{n} h\right) \leqq \mathrm{D}(h)$ as in II.1.2. Moreover:

$$
\mathrm{r}(h)=(\text { Cut, } A) \Rightarrow \mathrm{r}\left(h_{1}\right)=(\text { Cut }, A) \Rightarrow \ell(A)<\mathfrak{d}\left(h_{1}\right) \leqq \mathrm{d}(h) .
$$

III.1 $h=E h_{1}$ and $\mathrm{r}\left(h_{1}\right)=($ Cut, $C)$ : w.l.o.g. $C \in W_{0}-F O R$. Then $\mathfrak{r}(h)=\operatorname{Rep}_{0}$, $\mathfrak{s}_{n} h=R_{C}\left(E \mathfrak{s}_{1} h_{1}\right)\left(E \mathfrak{s}_{0} h_{1}\right)$ and (by I.H.) $\ell(C)<\mathrm{D}\left(h_{1}\right), \mathrm{D}\left(\mathfrak{s}_{i} h_{1}\right) \leqq \mathrm{D}\left(h_{1}\right)$ for $i=0,1$. Hence

$$
\begin{aligned}
\mathfrak{D}\left(\mathfrak{s}_{n} h\right) & =\max \left\{\ell(C), \mathrm{D}\left(E \mathfrak{s}_{1} h_{1}\right), \mathrm{D}\left(E \mathfrak{s}_{0} h_{1}\right)\right\} \\
& =\max \left\{\ell(C), \mathrm{D}\left(\mathfrak{s}_{1} h_{1}\right)-1, \mathrm{D}\left(\mathfrak{s}_{0} h_{1}\right)-1\right\} \\
& \left.\leqq \max \left\{\ell(C), \mathrm{D}\left(h_{1}\right)-1\right)\right\}=\mathrm{D}\left(h_{1}\right)-1=\mathrm{D}(h) .
\end{aligned}
$$

III. $h=E h_{1}$ and $\mathfrak{r}\left(h_{1}\right) \notin$ Cut: Then $\mathrm{r}(h)=\mathrm{r}\left(h_{1}\right), \mathfrak{s}_{n} h=E \mathfrak{s}_{n} h_{1}$, and (by I.H.) $\mathfrak{d}\left(\mathfrak{s}_{n} h_{1}\right) \leqq \mathfrak{d}\left(h_{1}\right)$. Hence

$$
\mathrm{D}\left(\mathfrak{s}_{n} h\right)=\mathrm{D}\left(\mathfrak{s}_{n} h_{1}\right)-1 \leqq \mathrm{D}\left(h_{1}\right)-1=\mathrm{D}(h) .
$$

c) By Remark 5.4b we have

(1) $\forall h \in H \forall n\left(\|\{h\}\| \in O n \&\left(\mathrm{r}(h) \neq A x \Rightarrow\left\|\left\{\mathfrak{s}_{n} h\right\}\right\|<\|\{h\}\|\right)\right)$.

We define $v(h) \in O n$, for $h \in H^{*}$, by recursion on $\ell^{*}(h)$ as follows:

$$
\begin{array}{cc}
v(h):=\|\{h\}\|, \quad \text { if } h \in H ; & v\left(I_{k, A} h_{1}\right):=v\left(h_{1}\right) ; \\
v\left(R_{C} h_{0} h_{1}\right):=v\left(h_{0}\right)+v\left(h_{1}\right) ; & v\left(E h_{1}\right):=\omega^{v\left(h_{1}\right)} .
\end{array}
$$

Using (1) one obtains by induction on $\ell^{*}(h)$ :

(2) $\forall h \in H^{*} \nabla n\left(x(h) \neq A x \Rightarrow v\left(s_{n} h\right)<v(h)\right)$ (cf. proof of $3.7 d$ ).

This gives

$$
\forall\left(n_{i}\right)_{i \in \mathbf{N}} \exists k\left(\{h\}\left(\left\langle n_{0}, \ldots, n_{k-1}\right\rangle\right)=q\left(\left[h,\left\langle n_{0}, \ldots, n_{k-1}\right\rangle\right]\right) \in \mathrm{Ax}\right),
$$

i.e. $\{h\} \in W T$. 
5.8 Theorem. If $\mathscr{H}$ (and thus also $\mathscr{H}^{*}$ ) is wellfounded then, for all $h_{0}, h_{1} \in H^{*}$, we have

$\left\{I_{k, A} h_{1}\right\}=\mathscr{I}_{k, A}\left(\left\{h_{1}\right\}\right),\left\{R_{\boldsymbol{C}} h_{0} h_{1}\right\}=\mathscr{R}_{\mathrm{C}}\left(\left\{h_{0}\right\},\left\{h_{1}\right\}\right),\left\{E h_{1}\right\}=\mathscr{E}\left(\left\{h_{1}\right\}\right)$.

Proof. We define v: $H^{*} \rightarrow W T$ by recursion on $\ell^{*}(h)$ (as in Sect. 3): $\mathbf{v}(h):=\{h\}$, if $h \in H ; \mathbf{v}\left(I_{k, A} h_{1}\right):=\mathscr{I}_{k, A}\left(\mathbf{v}\left(h_{1}\right)\right): \mathbf{v}\left(R_{C} h_{0} h_{1}\right):=\mathscr{R}_{C}\left(\mathbf{v}\left(h_{0}\right), \mathbf{v}\left(h_{1}\right)\right) ; \mathbf{v}\left(E h_{1}\right):=\mathscr{E}\left(\mathbf{v}\left(h_{1}\right)\right)$. By Theorem 5.3a) we have, for all $h \in H$,

$$
\mathbf{v}(h)(\langle\rangle)=\mathfrak{q}(h) \& \mathbf{v}\left(\mathfrak{s}_{n} h\right)=\mathbf{v}(h)[n] .
$$

But for $h \in H^{*} \backslash H$ we defined $\mathrm{e}(h), \mathfrak{r}(h), \mathfrak{s}_{n} h$ just in such a way that (*) also holds for all $h \in H^{*}$ (cf. 3.7, 5.5). So by 5.4a) we obtain $\mathbf{v}=\{\cdot\}$ and thus

$$
\left\{I_{k, A} h_{1}\right\}=\mathbf{v}\left(I_{k, A} h_{1}\right)=\mathscr{I}_{k, A}\left(\mathbf{v}\left(h_{1}\right)\right)=\mathscr{I}_{k, A}\left(\left\{h_{1}\right\}\right)
$$

etc.

\section{Continuous cut-elimination}

In this section we define an extension $\mathscr{E}^{\prime}: T R E E \rightarrow T R E E$ of $\mathscr{E}: W T \rightarrow W T$ such that $\forall \varphi \in T R E E\left(\varphi\right.$ locally correct $\Rightarrow \mathscr{E}^{\prime}(\varphi)$ locally correct $\left.\& \operatorname{deg}\left(\mathscr{E}^{\prime}(\varphi)\right) \leqq \operatorname{deg}(\varphi)-1\right)$. Moreover the functional $\lambda \varphi \lambda \sigma . \mathscr{E}^{\prime}(\varphi)(\sigma)$ will turn out primitive recursive so that $\mathscr{E}^{\prime}$ is automatically continuous. In fact we will prove a somewhat sharper result, namely

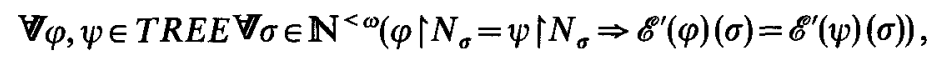

with

$$
N_{\left\langle n_{0}, \ldots, n_{k-1}\right\rangle}:=\left\{\left\langle m_{0}, \ldots, m_{\ell-1}\right\rangle: \ell \leqq k \&\left\{m_{0}, \ldots, m_{\ell-1}\right\} \leqq\left\{0,1, n_{0}, \ldots, n_{k-1}\right\}\right\} .
$$

6.1 Definition. $H:=\mathbb{N}^{<\omega}$ and, for each $\varphi \in T R E E, \mathscr{H}_{\varphi}:=(H, \varphi, \mathfrak{s})$ with $\mathfrak{s}: \mathbb{N} \times H \rightarrow H, \mathfrak{s}_{n}(\sigma):=\sigma *\langle n\rangle$. Obviously $\mathscr{H}_{\varphi}$ is a notation system. Let $\mathscr{H}_{\varphi}^{*}$ $=\left(H^{*}, \mathfrak{q}^{\varphi}, \mathfrak{s}^{\varphi}\right)$ be the $*$-extension of $\mathscr{H}_{\varphi}$ as defined in 5.5. Let $\{\cdot\}^{\varphi}: H^{*} \rightarrow T R E E$ be the canonical interpretation for $\mathscr{H}_{\varphi}^{*}$. Then we define

$$
\mathscr{E}^{\prime}: T R E E \rightarrow T R E E, \quad \mathscr{E}^{\prime}(\varphi):=\left\{E\langle>\}^{\varphi} .\right.
$$

6.2 Lemma. $\{\tau\}^{\varphi}=\varphi \llbracket \tau \rrbracket$, for each $\tau \in H$.

Proof. For every $\sigma=\left\langle n_{0}, \ldots, n_{k-1}\right\rangle \in H: \quad\{\tau\}^{\varphi}(\sigma)=\varphi([\tau, \sigma])=\varphi\left(\mathfrak{s}_{n_{k-1}} \ldots \mathfrak{s}_{n_{0}}(\tau)\right)$ $=\varphi(\tau * \sigma)=\varphi \llbracket \tau \rrbracket(\sigma)$.

6.3 Theorem. $\varphi \in W T \Rightarrow \mathscr{E}^{\prime}(\varphi)=\mathscr{E}(\varphi) \in W T$.

Proof. Using Lemma 6.2 and Theorem 5.8 we obtain: $\varphi \in W T \Rightarrow \forall \sigma\left(\{\sigma\}^{\varphi}\right.$ $=\varphi \llbracket \sigma \rrbracket \in W T) \Rightarrow \mathscr{H}_{\varphi}$ wellfounded $\Rightarrow \mathscr{E}^{\prime}(\varphi)=\{E\langle\rangle\}^{\varphi}=\mathscr{E}\left(\{\langle\rangle\}^{\varphi}\right)=\mathscr{E}(\varphi)$.

6.4 Theorem. $\varphi$ locally correct $\Rightarrow \mathscr{E}^{\prime}(\varphi)$ locally correct and $\operatorname{deg}\left(\mathscr{E}^{\prime}(\varphi)\right) \leqq \operatorname{deg}(\varphi)-1$.

Proof. By assumption we have $\forall \sigma . L C\left(\varphi(\sigma),\left(\varphi^{1}(\sigma *\langle n\rangle)\right)_{n \in \mathbb{N}}\right)$, i.e. $\mathscr{H}_{\varphi}$ is correct. Now by 5.7a) and 5.3b) we obtain that $\mathscr{H}_{\varphi}^{*}$ is correct and thus $\mathscr{E}^{\prime}(\varphi)=\{E<>\}^{\varphi}$ is 
locally correct. Let $m:=\operatorname{deg}(\varphi) \in \mathbb{N}$. Then $\mathrm{D}(h):=m$ is a cut-bound for $\mathscr{H}_{\varphi}$. By $\left.5.7 \mathrm{~b}\right)$ this can be extended to a cut-bound $\mathfrak{D}$ for $\mathscr{H}_{\varphi}^{*}$ with $\mathfrak{D}(E\langle\rangle)=\grave{D}(\langle\rangle)-1=m-1$. By 5.3c) we have $\operatorname{deg}\left(\mathscr{E}^{\prime}(\varphi)\right) \leqq D(E\langle>)$.

6.5. Remark. One easily verifies that the functions $q^{\varphi}: H^{*} \rightarrow R U L E \times S E Q$ and $\mathfrak{s}^{\boldsymbol{\varphi}}: \mathbb{N} \times H^{*} \rightarrow H^{*}$ are uniformly primitive recursive in $\varphi$. Together with $\mathscr{E}^{\prime}(\varphi)$ $\left(\left\langle n_{0}, \ldots, n_{k-1}\right\rangle\right)=\mathfrak{q}^{\varphi} \mathfrak{s}_{n_{k-1}}^{\varphi} \ldots \mathfrak{s}_{n_{0}}^{\varphi}(E\langle\rangle)$ this yields that the functional $(\varphi, \sigma) \mapsto \mathscr{E}^{\prime}(\varphi)(\sigma)$ is primitive recursive and therefore continuous, i.e. $\forall \varphi, \sigma \exists M \leqq \mathbb{N}^{<\omega}(M$ finite \& $\forall \psi\left(\varphi|M=\psi| M \Rightarrow \mathscr{E}^{\prime}(\varphi)(\sigma)=\mathscr{E}^{\prime}(\psi)(\sigma)\right)(*)$. In the following we establish (*) by a more direct argument. In fact we will prove more, namely

6.6 Theorem. $\forall \varphi, \psi \in T R E E \forall \sigma \in \mathbb{N}^{<\omega}\left(\varphi\left\lceil N_{\sigma}=\psi\left\lceil N_{\sigma} \Rightarrow \mathscr{E}^{\prime}(\varphi)(\sigma)=\mathscr{E}^{\prime}(\psi)(\sigma)\right)\right.\right.$, with the above defined $N_{\sigma}$.

Proof (informal). Let $\sigma=\left\langle n_{0}, \ldots, n_{k-1}\right\rangle$ and $\sigma_{i}:=\left\langle n_{0}, \ldots, n_{i-1}\right\rangle(i=0, \ldots, k)$. Then $\mathscr{E}^{\prime}(\varphi)(\sigma)=\mathfrak{q}^{\varphi} \mathfrak{s}_{n_{k}-1}^{\varphi} \ldots \mathfrak{s}_{n_{0}}^{\varphi}(E\langle\rangle)$. So $\mathscr{E}^{\prime}(\varphi)(\sigma)$ is obtained by computing successively $h_{1}:=\mathfrak{s}_{n_{0}}^{\varphi}(E\langle\rangle), h_{2}:=\mathfrak{s}_{n_{1}}^{\varphi} \mathfrak{s}_{n_{0}}^{\varphi}(E\langle\rangle), \ldots, h_{k}:=\mathfrak{s}_{n_{k}-1}^{\varphi} \ldots \mathfrak{s}_{n_{0}}^{\varphi}(E\langle\rangle)$, and $\mathfrak{q}^{\varphi}\left(h_{k}\right)$. - One easily verifies that for computing $\mathfrak{q}^{\varphi}(h)$ and $\mathfrak{s}_{n}^{\varphi}(h)\left(h \in H^{*}\right)$ one only needs to know $\varphi\left\lceil K(h)\right.$, where $K(h):=\{\sigma \in H: \sigma$ occurs in $h\}$. In addition $K\left(h_{i}\right) \subseteq N_{\sigma_{i}} \leqq N_{0}$ for $i=0, \ldots, k$. Hence for computing $\mathfrak{q}^{\varphi}\left(h_{k}\right)$ (i.e. $\left.\mathscr{E}^{\prime}(\varphi)(\sigma)\right)$ we only need to know $\varphi\left\lceil N_{\sigma}\right.$.

Formally the theorem is an immediate consequence of Lemmata $6.8,6.9$ below.

\subsection{Definitions}

(i) $M * N:=\{\sigma * \tau: \sigma \in M, \tau \in N\} \quad(M, N \subseteq H)$

(ii) $K(h):=\{\sigma \in H: \sigma$ occurs in $h\} \quad\left(h \in H^{*}\right)$

(iii) $\left[h,\left\langle n_{0}, \ldots, n_{k-1}\right\rangle\right]^{\varphi}:=\mathfrak{s}_{n_{k-1}}^{\varphi} \ldots s_{n_{0}}^{\varphi} h \quad\left(h \in H^{*}\right)$

\subsection{Lemma}

a) $h \in H^{*} \Rightarrow K\left(\mathfrak{s}_{n}^{\varphi} h\right) \subseteq K(h) * N_{\langle n\rangle}$

b) $h \in H^{*} \& \varphi\left\lceil K(h)=\omega\left\lceil K(h) \Rightarrow q^{\varphi}(h)=q^{\psi}(h) \& \mathfrak{s}_{n}^{\varphi} h=\mathfrak{s}_{n}^{\psi} h\right.\right.$.

Proof by induction on $\ell^{*}(h)$.

\subsection{Lemma}

a) $K\left(\left[E\langle>, \sigma]^{\varphi}\right) \cong N_{0}\right.$

b) $\varphi\left|N_{\sigma}=\psi\right| N_{\sigma} \Rightarrow[E\langle\rangle, \sigma]^{\varphi}=[E\langle\rangle, \sigma]^{\psi}$.

Proof by induction on length $(\sigma)$ using Lemma 6.8 .

\section{References}

1. Buchholz, W., Wainer, S.: Provably computable functions and the fast growing hierarchy. In: Simpsons, S.G. (ed.) Mathematical logic and combinatorics. AMS Contemp. Math. 65, 179-198 (1987)

2. Gentzen, G.: Die Widerspruchsfreiheit der reinen Zahlentheorie. Math. Ann. 112, 493-565 (1936)

3. Gentzen, G.: Neue Fassung des Widerspruchsfreiheitsbeweises für die reine Zahlentheorie. Forschungen zur Logik und zur Grundlegung der exakten Wissenschaften. Neue Folge 4, pp. 19-44 (1938)

4. Gentzen, G.: Beweisbarkeit und Unbeweisbarkeit von Anfangsfällen der transfiniten Induktion in der reinen Zahlentheorie. Math. Ann. 119, 149-161 (1943)

5. Girard, J.Y.: Proof theory and logical complexity. Studies in proof theory, Monographs 1. Napoli: Bibliopolis 1987 
6. Gordeev, L.: Proof-theoretical analysis: weak systems of functions and classes. Ann. Pure Appl. Logic 38, 1-121 (1988)

7. Kreisel, G.: Mathematical Logic. In: Saaty (ed.) Lectures on modern mathematics, vol. III, pp. 95-195. New York: Wiley 1965

8. Kreisel, G.: A survey of proof theory. JSL 33, 321-388 (1968)

9. Kreisel, G., Minc, G., Simpson, S.: The use of abstract language in elementary metamathematics: some pedagogic examples. In: Parikh, R. (ed.) Logic Colloqium, Boston. (Lect. Notes Math., vol. 453, pp. 38-131) Berlin Heidelberg New York: Springer 1975

10. Minc, G.: Finite investigations of transfinite derivations. J. Sov. Mat. 10(4), 548-596 (1978), (translated from: Zap. Nauchn. Semin. LOMI 49 (1975))

11. Smith, R.L.: The consistency strengths of some finite forms of the Higman and Kruskal theorems. In: Harrington, L.A. (ed.) Harvey Friedman's research on the foundations of mathematics, pp. 119-136. Amsterdam: North-Holland 1985

12. Schütte, K.: Beweistheoretische Erfassung der unendlichen Induktion in der Zahlentheorie. Math. Ann. 122, 369-389 (1951)

13. Schütte, K.: Beweistheorie. Berlin Heidelberg New York: Springer 1960

14. Schütte, K.: Proof Theory. Berlin Heidelberg New York: Springer 1977

15. Schwichtenberg, H.: Proof theory: some applications of cut-elimination. In: Barwise, J. (ed.) Handbook of Mathematical Logic. Amsterdam: North-Holland 1977

16. Schwichtenberg, H.: Ein einfaches Verfahren zur Normalisierung unendlicher Herleitungen. In: Börger, E. (ed.) Computation theory and logic. (Lect. Notes Comp. Sci., vol. 270, pp. 334-384) Berlin Heidelberg New York: Springer 1987

17. Tait, W.W.: Normal derivability in classical logic. In: Barwise, J. (ed.) The syntax and semantics of infinitary languages. (Lect. Notes Math., vol. 72, pp. 204-236) Berlin Heidelberg New York: Springer 1968

18. Takeuti, G.: Proof theory. 2nd edn. Amsterdam: North-Holland 1987 